\title{
THREE-DIMENSIONAL NUMERICAL LOCALIZATION OF IMPERFECTIONS BASED ON A LIMIT MODEL IN ELECTRIC FIELD AND A LIMIT PERTURBATION MODEL*
}

\author{
S.M. Mefire \\ LAMFA, CNRS UMR 6140, Université de Picardie, \\ 33 r. Saint-Leu, 80039 Amiens Cedex 1, France \\ Email: seraphin.mefire@u-picardie.fr
}

\begin{abstract}
From a limit model in electric field obtained by letting the frequency vanish in the timeharmonic Maxwell equations, we consider a limit perturbation model in the tangential boundary trace of the curl of the electric field for localizing numerically certain small electromagnetic inhomogeneities, in a three-dimensional bounded domain. We introduce here two localization procedures resulting from the combination of this limit perturbation model with each of the following inversion processes: the Current Projection method and an Inverse Fourier method. Each localization procedure uses, as data, a finite number of boundary measurements, and is employed in the single inhomogeneity case; only the one based on an Inverse Fourier method is required in the multiple inhomogeneities case. Our localization approach is numerically suitable for the context of inhomogeneities that are not purely electric. We compare the numerical results obtained from the two localization procedures in the single inhomogeneity configuration, and describe, in various settings of multiple inhomogeneities, the results provided by the procedure based on an Inverse Fourier method.

Mathematics subject classification: 65N21, 65N30, 78A25.

Key words: Inverse problems, Maxwell equations, Electric fields, Inhomogeneities, Electrical Impedance Tomography, Current Projection method, FFT, Numerical boundary measurements, Edge elements, Least square systems, Incomplete Modified Gram-Schmidt preconditioning.
\end{abstract}

\section{Introduction}

When we seek to localize an inhomogeneity of small volume contained in a three-dimensional bounded domain from a finite number of boundary measurements, we are usually concerned with an underlying inverse problem which is not in general well-posed. In the situation where the inverse problem is based on linear equations, the combination of an asymptotic expansion of the perturbation in the physical field in presence in the domain, with a suited inversion algorithm, can allow one to overcome the ill-posed character of this inverse problem. This is the approach proposed by Cedio-Fengya, Moskow \& Vogelius [10] for localizing a finite number of conductivity inhomogeneities, of small volume, contained in a bounded domain. Typically, the inversion algorithm makes use of an asymptotic expansion for perturbations in the voltage potential, and is based on a minimization process of least-squares type for the calculation of the geometrical parameters of the inhomogeneities. The resulting localization procedure is therefore iterative, in contrast to the procedure developed by Ammari, Moskow \& Vogelius [4]

\footnotetext{
* Received April 17, 2008 / Revised version received July 1, 2008 / Accepted October 20, 2008 /
} 
for such inhomogeneities. In fact, although using the same asymptotic expansion for measuring boundary voltage perturbations initiated by boundary electric currents, the inversion algorithm in [4] is based on one of the following two direct inversion processes: the Current Projection method and an Inverse Fourier method. In the first case, the algorithm consists of identifying the 'center' of a single inhomogeneity as the unknown of a linear system whereas in the second case, in the presence of multiple inhomogeneities, it consists of calculating a discrete inverse Fourier transform of a sample of measurements. Such direct processes appear numerically efficient (see e.g. $[4,22]$ ) for solving inverse problems where we are mainly interested in the position of the single inhomogeneity, or in the positions of a finite number of inhomogeneities, in the domain. We will be concerned with such processes in this paper. For other numerical methods that could be used in the localization problem of conductivity inhomogeneities, or of dielectric inhomogeneities, in different settings, we refer to $[2,8,12,15,16,20,21]$.

Recently, a framework for the localization of three-dimensional electromagnetic inhomogeneities was introduced by Ammari, Vogelius \& Volkov [5]. This framework considers the time-harmonic Maxwell equations in a three-dimensional bounded domain $\Omega$ containing a finite number $m$ of unknown inhomogeneities of small volume, and proposes to localize these inhomogeneities from an asymptotic expansion devoted to the study of perturbations in the tangential boundary trace of the curl of the electric field. A particular reformulation of this asymptotic expansion leads to an asymptotic formula that allows one to evaluate boundary measurements of "voltage" type from prescribed boundary currents. In the presence of well-separated inhomogeneities, and also distant from $\partial \Omega$, the boundary of $\Omega$, this asymptotic formula states that:

$$
\begin{aligned}
& \int_{\partial \Omega} \operatorname{curl} E_{\alpha} \times \nu \cdot w d \sigma-\int_{\partial \Omega} \operatorname{curl} w \times \nu \cdot\left(\nu \times\left(E_{\alpha} \times \nu\right)\right) d \sigma \\
& =\alpha^{3} \sum_{j=1}^{m} \omega^{2} \varepsilon_{0} \mu_{0}\left(\frac{\varepsilon_{0}}{\varepsilon_{j}}-1\right)\left[M^{j}\left(\frac{\varepsilon_{0}}{\varepsilon_{j}}\right) E_{0}\left(z_{j}\right)\right] \cdot w\left(z_{j}\right) \\
& +\alpha^{3} \sum_{j=1}^{m}\left(\frac{\mu_{0}}{\mu_{j}}-1\right)\left[M^{j}\left(\frac{\mu_{0}}{\mu_{j}}\right) \operatorname{curl} E_{0}\left(z_{j}\right)\right] \cdot \operatorname{curl} w\left(z_{j}\right)+\mathcal{O}\left(\alpha^{4}\right),
\end{aligned}
$$

where $w$ denotes any smooth vector-valued function such that

$$
\operatorname{curl}(\operatorname{curl} w)-k^{2} w=0 \text { in } W
$$

with $W$ an open neighborhood of $\Omega, k^{2}=\omega^{2} \varepsilon_{0} \mu_{0}$, and $\omega$ a given frequency. In (1.1), $\alpha$ is the common order of magnitude of the diameters of the inhomogeneities, and the points $z_{j}$, $1 \leq j \leq m$, represent the 'centers' of the inhomogeneities. The electric field is denoted by $E_{\alpha}$ in the presence of the inhomogeneities and by $E_{0}$ in the absence of all the inhomogeneities. The outward unit normal to $\Omega$, defined on $\partial \Omega$, is represented by $\nu$. The (constant) background magnetic permeability and complex permittivity are $\mu_{0}$ and $\varepsilon_{0}$ respectively. Also, $\mu_{j}$ and $\varepsilon_{j}$ are the (constant) magnetic permeability and the complex permittivity of the $j$ th inhomogeneity. Finally, $M^{j}\left(\mu_{0} / \mu_{j}\right)$ and $M^{j}\left(\varepsilon_{0} / \varepsilon_{j}\right)$ are the polarization tensors associated with the $j$ th inhomogeneity (symmetric $3 \times 3$ matrices).

More recently, Asch \& Mefire [7] have achieved in various contexts the numerical localization of such electromagnetic inhomogeneities from three numerical procedures based on (1.1). Typically, each of these procedures results from the combination of (1.1) with one of the following inversion processes: the Current Projection method, an Inverse Fourier method, and a MUSIC 
(MUltiple SIgnal Classification) algorithm [1, 15]. A numerical study of the asymptotic expansion for the perturbations in the tangential boundary trace of the curl of the electric field has been also achieved in [7]. It appears, in the single inhomogeneity case, and with respect to the parameter $\tau=\alpha \omega, \tau<1$, linking the order of magnitude of the diameter of the inhomogeneity and the frequency $\omega$, that this asymptotic expansion, and consequently (1.1), is numerically valid only within a restricted range of values of $\tau$. In the case of multiple inhomogeneities, the same remark comes from numerical simulations of their localization: in order to achieve accurate localizations, (1.1) must also be considered within a restricted range of values of $\tau$, where $\alpha$ represents now the common order of magnitude of the diameters of the inhomogeneities. In particular, the numerical investigations of [7] show, independently of each one of the used inversion processes, that the localization of these inhomogeneities is disastrous when the frequency $\omega$ becomes very small. Namely, for $\alpha / r<1$, where $r=\operatorname{diam}(\Omega) / 2$, these inhomogeneities cannot be localized from (1.1) in the context of very low frequencies. This observation leads us to an essential question.

Can we localize such inhomogeneities from the limit perturbation model obtained by considering purely and simply the frequency $\omega$ as equal to zero in the perturbation formula introduced in (1.1), and by using hence in the domain a limit model of the time-harmonic Maxwell equations resulting from this consideration?

This question is the motivation of the present paper, and its numerical treatment will constitute here our main work. We already mention that a positive numerical answer to this question would indicate a new numerical localization framework of electromagnetic inhomogeneities of small volume.

In this paper, we are concerned with an inverse problem that considers, in a three-dimensional bounded domain $\Omega$, the equations obtained by letting the frequency vanish in the time-harmonic Maxwell model formulated in electric field. As we shall see, these equations can be linked to the electrostatic system. Here also, $\Omega$ contains a finite number $m$ of unknown electromagnetic inhomogeneities of small volume and the inverse problem consists of localizing numerically these inhomogeneities from a finite number of measurements. This is an Electrical Impedance Tomography problem since the measurements are evaluated on the boundary of $\Omega$. This work can be connected to certain applications where it is sufficient to determine the positions of inhomogeneities without necessarily reconstructing their electromagnetic parameters or imaging their shapes in the identification process. In fact, our localization approach will mainly consist of locating the 'centers' of the inhomogeneities and in some situations, where $m=1$, of determining the 'center' and the diameter of the inhomogeneity. The framework developed in [5] is the basis of this localization approach, since we combine an analogous formula to (1.1) with a suited inversion algorithm. This analogous formula allows us to numerically compute boundary measurements of "voltage" type, and limited current-to-"voltage" pairs on the boundary of $\Omega$ are used as data of the inversion algorithm. Of course, as opposed to references concerned in the literature with the numerical localization, and using synthetic data, we think that the best way to check the robustness of a localization approach consists of using numerical data; each measurement will be thus evaluated here through finite element approximations.

This paper contains seven sections. We introduce in Section 2 some notation and briefly recall the framework developed in [5] for the localization of the inhomogeneities from a perturbation formula associated with the time-harmonic Maxwell equations. We describe in Section 3 a limit model in electric field, obtained from the time-harmonic Maxwell equations by letting the frequency vanish. Based on this limit model, we introduce the "background" problem in electric 
field as well as the problem in electric field in the presence of inhomogeneities in $\Omega$. We consider also in this section the formula for generating boundary measurements. This formula is obtained by letting the frequency vanish in the perturbation model associated with the time-harmonic Maxwell equations. In Section 4, we introduce a discrete formulation that combines Nédélec's edge elements with nodal finite elements. This formulation leads to a rectangular matrix system that allows us to compute the electric field (in the presence of inhomogeneities), initiated by a boundary current, for evaluating numerically the corresponding boundary measurement. The (direct) computation of the electric field, by a least squares approach, is performed a finite number of times equal to the finite number of electric currents to apply on the boundary of $\Omega$, in accordance with the localization procedure. We describe in Section 5, two localization procedures that will be used distinctly in the numerical simulations. Of course, combined with the formula for generating boundary measurements, the Current Projection method or an Inverse Fourier method allows us to define a numerical localization procedure. Any one of the two procedures can be employed for locating a single inhomogeneity, but only the one based on an Inverse Fourier method can be used for locating multiple inhomogeneities. In Section 6, we describe numerical results obtained, in various contexts, with each one of the two localization procedures. We are also interested in this section in a numerical comparison of the results of the two procedures in the single inhomogeneity case. We report finally, in Section 7, some conclusions.

\section{Preliminary Notation and a Perturbation Formula}

After introducing some notation and definitions, we consider in this part an asymptotic formula proposed by Ammari, Vogelius \& Volkov [5] for the study of perturbations in the electromagnetic fields due to the presence of small inhomogeneities in a bounded domain.

\subsection{Preliminary notation}

Let us consider a bounded open subset $\Omega$ of $\mathbb{R}^{3}$, that is convex. For simplicity we assume $\partial \Omega$, the boundary of $\Omega$, connected, to be $C^{\infty}$, but this regularity condition could be considerably weakened. The outward unit normal to $\Omega$, defined on $\partial \Omega$, is denoted by $\nu$. The domain $\Omega$ contains here a finite number $m$ of inhomogeneities, each one of the form $z_{j}+\alpha B_{j}$, where $B_{j} \subset \mathbb{R}^{3}$ is a bounded, smooth $\left(C^{\infty}\right)$ domain containing the origin. The total collection of inhomogeneities thus takes the form $I_{\alpha}=\bigcup_{j=1}^{m}\left(z_{j}+\alpha B_{j}\right)$. The points $z_{j} \in \Omega, 1 \leq j \leq m$, that determine the locations of the inhomogeneities are assumed to satisfy:

$$
\begin{cases}0<d_{0} \leq\left|z_{j}-z_{k}\right| & \forall j \neq k, \\ 0<d_{0} \leq \operatorname{dist}\left(z_{j}, \partial \Omega\right) \quad \forall j .\end{cases}
$$

The parameter $\alpha>0$, the common order of magnitude of the diameters of the inhomogeneities, is sufficiently small in such a way that these inhomogeneities are disjoint and their distance to $\mathbb{R}^{3} \backslash \Omega$ is larger than $d_{0} / 2$. As a consequence of the assumption (2.1), it follows that: $m \leq$ $6|\Omega| /\left(\pi d_{0}^{3}\right)$. As in [7], we call hereafter, an imperfection, each one of these small inhomogeneities.

When we study the time-harmonic solutions of linear Maxwell equations in $\Omega$ containing different materials, we consider $\mathbb{C}^{3}$-valued fields $E, H$ such that: $\forall x \in \Omega$,

$$
\operatorname{curl} E(x)=\mathrm{i} \omega \mu(x) H(x), \quad \operatorname{curl} H(x)=-\mathrm{i} \omega \varepsilon(x) E(x),
$$


where $\omega>0$ denotes the given frequency, $\mu$ is the magnetic permeability, $\varepsilon(x)=\varepsilon^{\mathrm{re}}(x)+$ $\mathrm{i} \sigma(x) / \omega$ represents the complex permittivity, with $\varepsilon^{\text {re }}$ the (real) electric permittivity and $\sigma$ the conductivity of the medium. By dividing the first equation of (2.2) by $\mu$ and taking the curl, we obtain the following equation for $E$ :

$$
\operatorname{curl}\left(\frac{1}{\mu} \operatorname{curl} E\right)-\omega^{2} \varepsilon E=0 \text { in } \Omega .
$$

Let $\mu_{0}>0, \varepsilon_{0}^{\text {re }}>0$, and $\sigma_{0} \geq 0$ denote the permeability, the (real) permittivity, and the conductivity of the background medium, with $\varepsilon_{0}=\varepsilon_{0}^{\text {re }}+\mathrm{i} \sigma_{0} / \omega$ the background complex permittivity. Let also $\mu_{j}>0, \varepsilon_{j}^{\text {re }}>0, \sigma_{j} \geq 0$ and $\varepsilon_{j}=\varepsilon_{j}^{\text {re }}+\mathrm{i} \sigma_{j} / \omega$ denote the permeability, the (real) permittivity, the conductivity, and the complex permittivity of the $j$ th imperfection $z_{j}+\alpha B_{j}$. For simplicity, we shall assume here that all these parameters are constants. Introduce thus the piecewise constant magnetic permeability $\mu_{\alpha}$ and the piecewise constant complex permittivity $\varepsilon_{\alpha}: \forall x \in \Omega$,

$$
\mu_{\alpha}(x)=\left\{\begin{array}{ll}
\mu_{0}, & \text { if } x \in \Omega \backslash \overline{I_{\alpha}}, \\
\mu_{j}, & \text { if } x \in z_{j}+\alpha B_{j},
\end{array} \quad \varepsilon_{\alpha}(x)= \begin{cases}\varepsilon_{0}, & \text { if } x \in \Omega \backslash \overline{I_{\alpha}}, \\
\varepsilon_{j}, & \text { if } x \in z_{j}+\alpha B_{j},\end{cases}\right.
$$

with $1 \leq j \leq m$. If we allow the degenerate case $\alpha=0$, then the function $\mu_{\alpha}$ equals the constant $\mu_{0}$ and the function $\varepsilon_{\alpha}$ equals the constant $\varepsilon_{0}$.

Let $\left\{\gamma_{n}\right\}_{0 \leq n \leq m}$ be a set of complex constants with $\operatorname{Re}\left(\gamma_{n}\right)>0$, for $0 \leq n \leq m$. Typically, $\left\{\gamma_{n}\right\}_{0 \leq n \leq m}$ will be related to either the set $\left\{\mu_{n}\right\}_{0 \leq n \leq m}$ or the set $\left\{\varepsilon_{n}\right\}_{0 \leq n \leq m}$. For any fixed $1 \leq j_{0} \leq m$, let $\gamma$ denote the function defined as: $\forall x \in \mathbb{R}^{3}$,

$$
\gamma(x)=\left\{\begin{array}{lll}
\gamma_{0}, & \text { if } & x \in \mathbb{R}^{3} \backslash \overline{B_{j_{0}}}, \\
\gamma_{j_{0}}, & \text { if } & x \in B_{j_{0}}
\end{array}\right.
$$

Let $1 \leq l \leq 3$. We denote by $\phi_{l}$ the solution to the problem:

$$
\left\{\begin{array}{rll}
\operatorname{div}\left(\gamma(x) \operatorname{grad} \phi_{l}(x)\right)=0 & \text { for } & x \in \mathbb{R}^{3} \\
\phi_{l}(x)-x_{l} \rightarrow 0 & \text { as } & |x| \rightarrow \infty
\end{array}\right.
$$

As mentioned in [5], the existence and uniqueness of $\phi_{l}$ can be established (in the real as well as in the complex case) by using single layer potentials with suitably chosen densities ([11, 13]). When the outward unit normal to $B_{j_{0}}$ defined on $\partial B_{j_{0}}$, the boundary of $B_{j_{0}}$, is also denoted by $\nu$, and the superscripts,+- indicate the limiting values as $\partial B_{j_{0}}$ is approached from outside $B_{j_{0}}$, and from inside $B_{j_{0}}$ respectively, this problem in scalar potential may also be reformulated as follows:

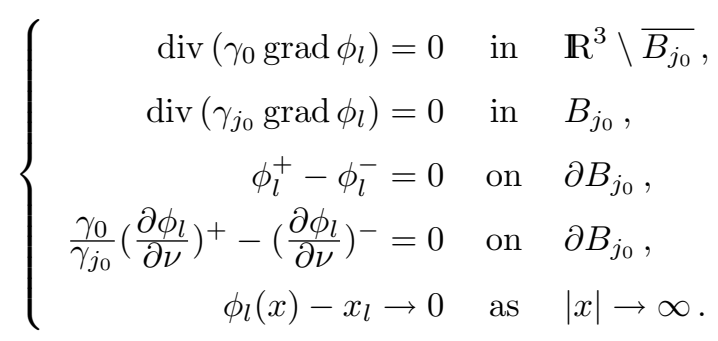

The function $\phi_{l}$ depends thus only on $\gamma_{0}$ and $\gamma_{j_{0}}$ through the ratio $c=\gamma_{0} / \gamma_{j_{0}}$. Here, the essential assumption is that the constant $c$ cannot be zero or a negative real number. With this 
aspect ratio, we define (as in [5]) the polarization tensor, $M^{j_{0}}(c)$, of the inhomogeneity $B_{j_{0}}$ as follows: $\forall 1 \leq i, l \leq 3$,

$$
M_{i l}^{j_{0}}(c)=c^{-1} \int_{B_{j_{0}}} \frac{\partial \phi_{l}}{\partial x_{i}} d x .
$$

Following [5], the tensor $M^{j_{0}}(c)$ is symmetric, and is furthermore positive definite if $c \in \mathbb{R}_{+}^{\star}$.

\subsection{A perturbation formula}

Each vector field subject to (2.3) will be called hereafter the "electric field". In the presence of imperfections, the electric field denoted $E_{\alpha}$ is the solution to:

$$
\left\{\begin{aligned}
\operatorname{curl}\left(\frac{1}{\mu_{\alpha}} \operatorname{curl} E_{\alpha}\right)-\omega^{2} \varepsilon_{\alpha} E_{\alpha}=0 & \text { in } \quad \Omega, \\
E_{\alpha} \times \nu=g & \text { on } \partial \Omega,
\end{aligned}\right.
$$

with $g$ a given datum on $\partial \Omega$. In the absence of all the imperfections, the electric field denoted $E_{0}$ satisfies:

$$
\left\{\begin{aligned}
\operatorname{curl}\left(\frac{1}{\mu_{0}} \operatorname{curl} E_{0}\right)-\omega^{2} \varepsilon_{0} E_{0}=0 & \text { in } \quad \Omega, \\
E_{0} \times \nu=g & \text { on } \quad \partial \Omega .
\end{aligned}\right.
$$

Let

$$
H(\operatorname{curl} ; \Omega)=\left\{u \in\left(L^{2}(\Omega)\right)^{3} ; \operatorname{curl} u \in\left(L^{2}(\Omega)\right)^{3}\right\}
$$

be endowed with its usual Hermitian product denoted here by $(., .)_{H(\operatorname{curl} ; \Omega)}$; the corresponding norm is denoted by $\|$. $\|_{H(\operatorname{curl} ; \Omega)}$. By representing the surface divergence by div $\partial \Omega$, let us consider the space

$$
T H^{-\frac{1}{2}}(\operatorname{div} ; \partial \Omega)=\left\{q \in\left(H^{-\frac{1}{2}}(\partial \Omega)\right)^{3} ; \operatorname{div}_{\partial \Omega} q \in H^{-\frac{1}{2}}(\partial \Omega), q \cdot \nu=0 \text { on } \partial \Omega\right\},
$$

with its usual norm denoted here by $\|\cdot\|_{T H^{-\frac{1}{2}}(\operatorname{div} ; \partial \Omega)}$.

Of course, the vector fields $E_{\alpha}$ and $E_{0}$, satisfying (2.6) and (2.7) respectively, are determined in $H(\operatorname{curl} ; \Omega)$, and the datum $g$ is taken in $T H^{-\frac{1}{2}}(\operatorname{div} ; \partial \Omega)$.

We will be interested in an interpretation of the following statement, obtained from the framework developed by Ammari, Vogelius \& Volkov [5], and that introduces in particular a version of the boundary perturbation in the curl of the electric field due to the presence of imperfections.

Theorem 2.1. Let (2.1) be satisfied, and $k^{2}=\omega^{2} \mu_{0} \varepsilon_{0}$ be taken such that the natural weak formulation associated with (2.7) has a unique solution. Let us denote by $w$ any smooth vectorvalued function such that:

$$
\operatorname{curl}(\operatorname{curl} w)-k^{2} w=0 \quad \text { in } W,
$$

where $W$ is an open neighborhood of $\Omega$. There exists a constant $\alpha_{0}>0$ such that, for a given $g \in T H^{-\frac{1}{2}}(\operatorname{div} ; \partial \Omega)$ and any $0<\alpha<\alpha_{0}$, the boundary value problem (2.6) has a unique (weak) solution. The constant $\alpha_{0}$ depends on $\left\{B_{j}\right\}_{1 \leq j \leq m}, \Omega,\left\{\mu_{j}\right\}_{0 \leq j \leq m},\left\{\varepsilon_{j}\right\}_{0 \leq j \leq m}, \omega$, and $d_{0}$, but is otherwise independent of $w$, and of the points $z_{j}, 1 \leq j \leq m$. Moreover, the physical 
fields $E_{\alpha}$ and $E_{0}$ satisfy:

$$
\begin{aligned}
& \int_{\partial \Omega} \operatorname{curl} E_{\alpha} \times \nu \cdot w d \sigma-\int_{\partial \Omega} \operatorname{curl} w \times \nu \cdot\left(\nu \times\left(E_{\alpha} \times \nu\right)\right) d \sigma \\
& =\alpha^{3} \sum_{j=1}^{m} \omega^{2} \varepsilon_{0} \mu_{0}\left(\frac{\varepsilon_{0}}{\varepsilon_{j}}-1\right)\left[M^{j}\left(\frac{\varepsilon_{0}}{\varepsilon_{j}}\right) E_{0}\left(z_{j}\right)\right] \cdot w\left(z_{j}\right) \\
& +\alpha^{3} \sum_{j=1}^{m}\left(\frac{\mu_{0}}{\mu_{j}}-1\right)\left[M^{j}\left(\frac{\mu_{0}}{\mu_{j}}\right) \operatorname{curl} E_{0}\left(z_{j}\right)\right] \cdot \operatorname{curl} w\left(z_{j}\right)+\mathcal{O}\left(\alpha^{4}\right) .
\end{aligned}
$$

As now known from [7], where the numerical localization of imperfections using (2.6) - (2.7) is performed in various contexts, while combined with an inversion process, the statement of this theorem constitutes actually an efficient localization approach.

\section{The Limit Model in Electric Field and the Perturbation Model}

When we consider the case where the conductivity vanishes everywhere in $\Omega$, we define of course a real-valued electric permittivity everywhere in $\Omega: \varepsilon \equiv \varepsilon^{\mathrm{re}}$. By reducing on the other hand the frequency $\omega$ until its limiting value in order to take $\omega$ as equal to zero in (2.3), we are concerned in fact with the system which consists of finding $E$ such that:

$$
\left\{\begin{aligned}
\operatorname{curl}\left(\frac{1}{\mu} \operatorname{curl} E\right)=0 & \text { in } \quad \Omega, \\
\operatorname{div}(\varepsilon E)=0 & \text { in } \quad \Omega .
\end{aligned}\right.
$$

Contrary to the model introduced in (2.3), the divergence-free constraint is here explicitly taken into account. The present model can be connected to the system of electrostatics in the sense that any vector field satisfying the equations of electrostatics with a zero charge density also verifies (3.1). Non-trivial boundary conditions for $E \times \nu$, on the boundary $\partial \Omega$, shall be prescribed in order to arrive at particular non-trivial solutions to (3.1). Let us recall $\mu_{\alpha}$ the piecewise constant permeability, and $\varepsilon_{\alpha}$ the piecewise constant permittivity then now real-valued.

The electric field denoted $E_{\alpha}$, in the presence of imperfections, satisfies:

$$
\left\{\begin{array}{rll}
\operatorname{curl}\left(\frac{1}{\mu_{\alpha}} \operatorname{curl} E_{\alpha}\right)=0 & \text { in } & \Omega, \\
\operatorname{div}\left(\varepsilon_{\alpha} E_{\alpha}\right)=0 & \text { in } \quad & \Omega, \\
E_{\alpha} \times \nu=g & \text { on } & \partial \Omega,
\end{array}\right.
$$

where $g$ is a given datum on $\partial \Omega$.

The electric field denoted $E_{0}$, in the absence of all the imperfections, is such that

$$
\left\{\begin{array}{rll}
\operatorname{curl}\left(\frac{1}{\mu_{0}} \operatorname{curl} E_{0}\right)=0 & \text { in } & \Omega, \\
\operatorname{div}\left(\varepsilon_{0} E_{0}\right)=0 & \text { in } & \Omega, \\
E_{0} \times \nu=g & \text { on } & \partial \Omega .
\end{array}\right.
$$

The fields $E_{\alpha}$ and $E_{0}$ will be sought in $H(\operatorname{curl} ; \Omega)$, and the datum $g$ used in (3.2) and (3.3) will be taken in $T H^{-\frac{1}{2}}(\operatorname{div} ; \partial \Omega)$. For such a datum $g$, let us consider $u_{g} \in H(\operatorname{curl} ; \Omega)$ such 
that (see, e.g., [6]):

$$
\left\{\begin{array}{l}
u_{g} \times \nu=g \text { on } \partial \Omega \\
\left\|u_{g}\right\|_{H(\operatorname{curl} ; \Omega)} \leq C_{\Omega}\|g\|_{T H^{-\frac{1}{2}}(\operatorname{div} ; \partial \Omega)}
\end{array}\right.
$$

where $C_{\Omega}>0$ is a constant depending only on $\Omega$. With this extension field, the determination of $E_{\alpha}$ satisfying (3.2) is reduced to the problem which consists of finding $\mathcal{E}_{\alpha}$ such that:

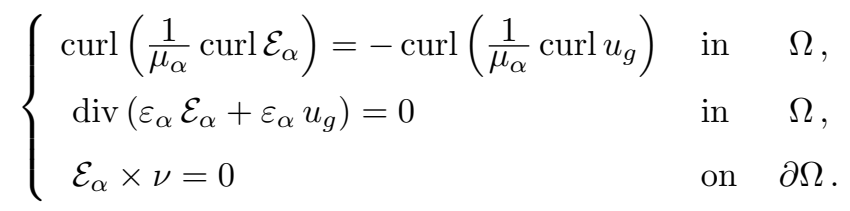

Of course, knowing $u_{g}$, while $\mathcal{E}_{\alpha}$ is in accordance with (3.5), we determine the electric field:

$$
E_{\alpha}:=\mathcal{E}_{\alpha}+u_{g}
$$

A similar reformulation can be applied to the system (3.3) verified by $E_{0}$, by using the same extension field. Let us set:

$$
\Psi=H_{0}^{1}(\Omega), \quad \mathcal{H}=\{u \in H(\operatorname{curl} ; \Omega) ; u \times \nu=0 \text { on } \partial \Omega\} .
$$

The vector field $\mathcal{E}_{\alpha}$ is sought in the space $\mathcal{H}$. From the field $u_{g}$, let us consider $q \in \Psi$ such that

$$
\left(\varepsilon_{\alpha} \operatorname{grad} q, \operatorname{grad} \psi\right)_{\left(L^{2}(\Omega)\right)^{3}}=\left(\varepsilon_{\alpha} u_{g}, \operatorname{grad} \psi\right)_{\left(L^{2}(\Omega)\right)^{3}} \quad \forall \psi \in \Psi .
$$

Typically,

$$
\|\operatorname{grad} q\|_{\left(L^{2}(\Omega)\right)^{3}} \leq C\left\|u_{g}\right\|_{\left(L^{2}(\Omega)\right)^{3}},
$$

with $C>0$ a constant depending on $\max \left\{\varepsilon_{n} ; 0 \leq n \leq m\right\}$ and $\min \left\{\varepsilon_{n} ; 0 \leq n \leq m\right\}$. Here, $(., .)_{\left(L^{2}(\Omega)\right)^{3}}$ represents the usual Hermitian product of $\left(L^{2}(\Omega)\right)^{3}$, and $\|\cdot\|_{\left(L^{2}(\Omega)\right)^{3}}$ denotes the associated norm. By writing then, with the aid of this scalar potential, the second equation of (3.5) in the weak form,

$$
\left(\varepsilon_{\alpha} \mathcal{E}_{\alpha}+\varepsilon_{\alpha} \operatorname{grad} q, \operatorname{grad} \psi\right)_{\left(L^{2}(\Omega)\right)^{3}}=0 \quad \forall \psi \in \Psi
$$

and setting:

$$
U_{\alpha}:=\mathcal{E}_{\alpha}+\operatorname{grad} q
$$

it follows that

$$
\left(\varepsilon_{\alpha} U_{\alpha}, \operatorname{grad} \psi\right)_{\left(L^{2}(\Omega)\right)^{3}}=0 \quad \forall \psi \in \Psi .
$$

The new unknown $U_{\alpha}$ is on the other hand such that: $U_{\alpha} \times \nu=\mathcal{E}_{\alpha} \times \nu=0$ on $\partial \Omega$. Let us set:

$$
\mathcal{V}=\left\{v \in H(\operatorname{curl} ; \Omega) ;\left(\varepsilon_{\alpha} v, \operatorname{grad} \psi\right)_{\left(L^{2}(\Omega)\right)^{3}}=0 \quad \forall \psi \in \Psi, v \times \nu=0 \text { on } \partial \Omega\right\} .
$$

Due to the definition of the real-valued parameter $\varepsilon_{\alpha}$ and to hypotheses on the geometry of $\Omega$, the mapping $u \in \mathcal{V} \longmapsto\|\operatorname{curl} u\|_{\left(L^{2}(\Omega)\right)^{3}}$ generates (see, e.g., [6]) a norm that is equivalent to $\|.\|_{H(\operatorname{curl} ; \Omega)}$. We endow the space $\mathcal{V}$ with this generated norm.

By inserting into the first equation of (3.5) the expression of $\mathcal{E}_{\alpha}$ given in (3.8), we obtain a new equation satisfied by $U_{\alpha}$ that allows us to introduce the weak formulation defined below for $g \in T H^{-\frac{1}{2}}(\operatorname{div} ; \partial \Omega)$ and therefore for $u_{g}$ taken as in (3.4). 
Find $U_{\alpha} \in \mathcal{V}$ such that:

$$
\left(\frac{1}{\mu_{\alpha}} \operatorname{curl} U_{\alpha}, \operatorname{curl} v\right)_{\left(L^{2}(\Omega)\right)^{3}}=-\left(\frac{1}{\mu_{\alpha}} \operatorname{curl} u_{g}, \operatorname{curl} v\right)_{\left(L^{2}(\Omega)\right)^{3}} \quad \forall v \in \mathcal{V} .
$$

It is already important to specify that the existence and the uniqueness of such a vector field $U_{\alpha}$ will lead to the determination of a unique vector field $\mathcal{E}_{\alpha}$ in accordance with (3.5), due to the definition of the scalar potential $q$ in (3.8). Consequently, the existence and the uniqueness of $E_{\alpha}$ satisfying (3.2) will be ensured by taking into account (3.6).

Remark 3.1. Let us mention that it was possible to introduce a weak formulation such as the one defined in (3.9) but with $\mathcal{E}_{\alpha}$ as the unknown, by considering therefore in (3.4) the extension field $u_{g}$ under the requirement: $\operatorname{div}\left(\varepsilon_{\alpha} u_{g}\right)=0$ in $\Omega$. However, according to the heterogeneity of the domain, this requirement would complicate the determination of an analytic expression of $u_{g}$, in fact necessary in the numerical approximations. As we shall observe in the section regarding applications, each choice of the datum $g$ will directly provide the analytic expression of an associated extension field, not subject to the mentioned requirement. We will be concerned (in Section 4) with a discrete formulation that requires $u_{g}$ for numerically approximating $\mathcal{E}_{\alpha}$ (and therefore $E_{\alpha}$ ), but where the scalar potential $q$ does not intervene.

In the following statement, we are interested in the existence and the uniqueness of the solution of the weak formulation introduced here.

Theorem 3.1. For any $g \in T H^{-\frac{1}{2}}(\operatorname{div} ; \partial \Omega)$, and therefore any $u_{g}$ defined as in (3.4), the formulation (3.9) has one and only one solution $U_{\alpha} \in \mathcal{V}$. Furthermore, there exists a constant $C>0$ independent of $\alpha$ such that: $\left\|U_{\alpha}\right\|_{H(\operatorname{curl} ; \Omega)} \leq C\|g\|_{T H^{-\frac{1}{2}}(\operatorname{div} ; \partial \Omega)}$.

Proof. Let $g \in T H^{-\frac{1}{2}}(\operatorname{div} ; \partial \Omega)$, and $u_{g}$ be defined as in (3.4). Denoting by $a_{\alpha}$ the sesquilinear form such that $a_{\alpha}(u, v)=\left(\frac{1}{\mu_{\alpha}} \operatorname{curl} u, \operatorname{curl} v\right)_{\left(L^{2}(\Omega)\right)^{3}}$, and by $l_{\alpha}$ the functional such that $l_{\alpha}(v)=-\left(\frac{1}{\mu_{\alpha}} \operatorname{curl} u_{g}, \operatorname{curl} v\right)_{\left(L^{2}(\Omega)\right)^{3}}$ for $u, v \in \mathcal{V}$, the weak equation in (3.9) becomes: $a_{\alpha}\left(U_{\alpha}, v\right)=l_{\alpha}(v) \forall v \in \mathcal{V}$. According to the equivalence of the norms of $\mathcal{V}$ and $H(\operatorname{curl} ; \Omega)$, and with the definitions of $\mu_{\alpha}, \varepsilon_{\alpha}$, it follows that $a_{\alpha}$ is continuous on $\mathcal{V} \times \mathcal{V}$ and is $\mathcal{V}$-elliptic; it also results that $l_{\alpha}$ is continuous on $\mathcal{V}$. The Lax-Milgram Theorem allows us then to conclude that (3.9) has a unique solution $U_{\alpha} \in \mathcal{V}$ satisfying the specified estimate.

Although $E_{\alpha}$ and $E_{0}$ have been only defined in a weak sense on $\partial \Omega$, elliptic regularity results ensure that the term $\left(\frac{1}{\mu_{\alpha}} \operatorname{curl} E_{\alpha}-\frac{1}{\mu_{0}} \operatorname{curl} E_{0}\right) \times\left.\nu\right|_{\partial \Omega}$ is infinitely smooth (when $g$ is infinitely smooth). We are hereafter interested in a version of this perturbation term, and more precisely in an interpretation of the asymptotic formula of Theorem 2.1, in the context of the limit model introduced in this section.

Remark 3.2. By considering the framework developed by Ammari, Vogelius \& Volkov [5], where we take the frequency $\omega$ as equal to zero, we obtain an approximation of the boundary perturbation in the curl of the electric field associated with the present model. Of course, since the remainder term in (2.8) can be bounded by $C \alpha^{4}$, with $C>0$ a constant depending (in particular) on certain non negative powers of $\omega$ (see e.g. [3]), we first neglect in (2.8) this asymptotically small remainder term, and next let $\omega$ vanish in the resulting formula. We are 
thus concerned with a limit perturbation model, based on the following approximation

$$
\begin{aligned}
& \int_{\partial \Omega} \operatorname{curl} E_{\alpha} \times \nu \cdot w d \sigma-\int_{\partial \Omega} \operatorname{curl} w \times \nu \cdot\left(\nu \times\left(E_{\alpha} \times \nu\right)\right) d \sigma \\
& \approx \alpha^{3} \sum_{j=1}^{m}\left(\frac{\mu_{0}}{\mu_{j}}-1\right)\left[M^{j}\left(\frac{\mu_{0}}{\mu_{j}}\right) \operatorname{curl} E_{0}\left(z_{j}\right)\right] \cdot \operatorname{curl} w\left(z_{j}\right),
\end{aligned}
$$

where the fields $E_{\alpha}$ and $E_{0}$ are subject respectively to (3.2) and (3.3) with the datum $g$ considered in $T H^{-\frac{1}{2}}(\operatorname{div} ; \partial \Omega)$. Now, $w$ is any smooth vector-valued function such that:

$$
\left\{\begin{aligned}
\operatorname{curl}\left(\frac{1}{\mu_{0}} \operatorname{curl} w\right)=0 & \text { in } \quad W, \\
\operatorname{div}\left(\varepsilon_{0} w\right)=0 & \text { in } \quad W,
\end{aligned}\right.
$$

where $W$ is an open neighborhood of $\Omega$. Of course, here also $0<\alpha<\alpha_{0}$ with the constant $\alpha_{0}>0$ independent of the points $z_{j}, 1 \leq j \leq m$, and of the function $w$.

The approximation formula (3.10) appears well suited to applications since as we shall see in Section 5, it allows us to effectively localize imperfections by some special choices of $w$ and by the use of particular inversion algorithms.

Remark 3.3. Let us already mention however that, since only explicit information on the parameter $\mu_{\alpha}$ appears in (3.10), as long as the imperfections are purely electric, their localization cannot be determined from this formula.

\section{Numerical Approximations}

By making use of finite elements, we will discretize (3.9) and then introduce a discrete formulation that allows us to numerically approximate the vector field $\mathcal{E}_{\alpha}$ (and hence to approximate the electric field $E_{\alpha}$ ).

\subsection{Preliminaries}

In order to simplify the presentation, we assume, in this section and in the following ones, that each imperfection present in the domain is a polyhedron, as well as the domain. In view of the numerical localizations, we will use the formula (3.10) and therefore the discrete field associated with the electric field $E_{\alpha}$. This discrete field will be obtained from a finite element method based on a mesh obtained by a usual process of triangulation of the domain. Typically, the conforming finite element triangulation $\mathcal{T}_{\alpha}$ of $\Omega$ is made up of tetrahedra in such a way that each inhomogeneity corresponds to a distinct collection of tetrahedra of $\mathcal{T}_{\alpha}$. More precisely, the collection of tetrahedra associated with an inhomogeneity covers entirely the geometry of the inhomogeneity. With such a conforming mesh of $\Omega$, we are able to introduce a discrete formulation whose matrix assembly is easily performed by decomposing each heterogeneous integral term of the formulation into a sum of homogeneous integral terms.

For a tetrahedron $K$, let us denote by $\varrho_{K}$ the diameter of the largest sphere included in $K$, and by $h_{K}$ the diameter of $K$. The aspect-ratio of $\mathcal{T}_{\alpha}$ is defined as follows: $h_{\mathcal{T}_{\alpha}}=\sup _{K \in \mathcal{T}_{\alpha}} h_{K}$. Let us call $\left\{\mathcal{T}_{\alpha, n}\right\}_{n \geq 1}$ a sequence of triangulations of the domain $\Omega$, where $\mathcal{T}_{\alpha, n}$ is described as 
$\mathcal{T}_{\alpha}$ above, for each $n$. As usual, we assume that this sequence is regular in the sense that there exists a constant $c>0$ such that:

$$
\forall n, \sup _{K \in \mathcal{T}_{\alpha, n}} \frac{h_{K}}{\varrho_{K}} \leq c
$$

and moreover,

$$
\lim _{n \longrightarrow \infty} h_{\mathcal{T}_{\alpha, n}}=0
$$

Let $n \geq 1$ be fixed. It is important to notice the dependence of $h_{\mathcal{T}_{\alpha, n}}$ on $\alpha$ in the sense that we need to have a triangulation $\mathcal{T}_{\alpha, n}$ of $\Omega$ as fine as the triangulation of the smallest imperfection. In the sequel, we denote $\mathcal{T}_{\alpha}$ instead of $\mathcal{T}_{\alpha, n}$ and $h$ instead of $h_{\mathcal{T}_{\alpha, n}}$ when no confusion is possible.

\subsection{Discrete formulation in electric field}

We start by discretizing (3.9) with the help of the edge elements (see Nédélec [18]) of the first order, in particular. By denoting by $K$ a tetrahedron of $\mathcal{T}_{\alpha}$, let us consider

$$
R^{1}(K)=\left\{u: K \longrightarrow \mathbb{C}^{3} ; \exists a, b \in \mathbb{C}^{3}, u(x)=a+b \times x, x=\left(x_{1}, x_{2}, x_{3}\right)^{T} \in K\right\} .
$$

The superscript "T" denotes, here and in the next sections, the transpose. Let us set

$$
\mathcal{R}_{h}=\left\{u_{h} \in H(\operatorname{curl} ; \Omega) ;\left.u_{h}\right|_{K} \in R^{1}(K) \forall K \in \mathcal{T}_{\alpha}\right\},
$$

and associate with $\mathcal{H}$ the discrete space

$$
\mathcal{H}_{h}=\left\{u_{h} \in \mathcal{R}_{h} ; u_{h} \times \nu=0 \text { on } \partial \Omega\right\} .
$$

Of course, as in the case of $\mathcal{H}$, the discrete space $\mathcal{H}_{h}$ is also endowed with the Hermitian product of $H(\operatorname{curl} ; \Omega)$.

In view of a practical implementation, the expression of any vector field of $\mathcal{R}_{h}$ in each tetrahedron $K \in \mathcal{T}_{\alpha}$ can be written with the help of barycentric coordinates associated with $K$, similarly as done in [17] for $\mathbb{R}^{3}$-valued fields.

We associate with $\Psi$ the discrete space

$$
\Psi_{h}=\left\{\psi_{h} \in H^{1}(\Omega) ;\left.\operatorname{Re}\left(\psi_{h}\right)\right|_{K},\left.\operatorname{Im}\left(\psi_{h}\right)\right|_{K} \in P_{1} \quad \forall K \in \mathcal{T}_{\alpha}, \psi_{h}=0 \text { on } \partial \Omega\right\},
$$

where $P_{1}$ is the space of polynomials of degree less than or equal to 1 . By setting then

$$
\mathcal{V}_{h}=\left\{v_{h} \in \mathcal{R}_{h} ;\left(\varepsilon_{\alpha} v_{h}, \operatorname{grad} \psi_{h}\right)_{\left(L^{2}(\Omega)\right)^{3}}=0 \quad \forall \psi_{h} \in \Psi_{h}, v_{h} \times \nu=0 \text { on } \partial \Omega\right\},
$$

we define of course the discrete space associated with $\mathcal{V}$, and endowed with the norm of $\mathcal{V}$. We can now introduce the discrete formulation associated with (3.9) as follows.

Find $U_{h} \in \mathcal{V}_{h}$ such that:

$$
\left(\frac{1}{\mu_{\alpha}} \operatorname{curl} U_{h}, \operatorname{curl} v_{h}\right)_{\left(L^{2}(\Omega)\right)^{3}}=-\left(\frac{1}{\mu_{\alpha}} \operatorname{curl} u_{g}, \operatorname{curl} v_{h}\right)_{\left(L^{2}(\Omega)\right)^{3}} \quad \forall v_{h} \in \mathcal{V}_{h} .
$$

Due to the conforming finite element method applied here, and in particular to properties (see, e.g., Theorem 1 in [19]) of the considered finite elements, according to the definitions of $\mu_{\alpha}, \varepsilon_{\alpha}$, as well to the assumptions on the geometry of $\Omega$, it follows that (4.1) has a unique solution. 
Although well-posed, this formulation is not well suited to a flexible numerical implementation because of the divergence-free constraint that must be treated implicitly. Let us then rewrite (4.1) as bellow.

Find $U_{h} \in \mathcal{H}_{h}$ such that:

$$
\left\{\begin{array}{l}
\left(\frac{1}{\mu_{\alpha}} \operatorname{curl} U_{h}, \operatorname{curl} v_{h}\right)_{\left(L^{2}(\Omega)\right)^{3}}=-\left(\frac{1}{\mu_{\alpha}} \operatorname{curl} u_{g}, \operatorname{curl} v_{h}\right)_{\left(L^{2}(\Omega)\right)^{3}} \quad \forall v_{h} \in \mathcal{H}_{h}, \\
\left(\varepsilon_{\alpha} U_{h}, \operatorname{grad} \psi_{h}\right)_{\left(L^{2}(\Omega)\right)^{3}}=0 \quad \forall \psi_{h} \in \Psi_{h} .
\end{array}\right.
$$

The divergence-free constraint is now explicitly taken into account, and (4.2) is more suitable than (4.1) for a flexible implementation. By using then $U_{h}$ as an approximation of the vector field $U_{\alpha}$ satisfying (3.9), let us introduce, following (3.8),

$$
U_{h}=: \mathcal{E}_{h}+\operatorname{grad} q
$$

the vector field $\mathcal{E}_{h}$ as the discrete field associated with $\mathcal{E}_{\alpha}$ subject to (3.5). By inserting this expression of $U_{h}$ into (4.2), and taking into account the definition of the scalar potential $q$ from (3.7), we introduce the following discrete formulation.

Find $\mathcal{E}_{h} \in \mathcal{H}_{h}$ such that:

$$
\left\{\begin{array}{l}
\left(\frac{1}{\mu_{\alpha}} \operatorname{curl} \mathcal{E}_{h}, \operatorname{curl} v_{h}\right)_{\left(L^{2}(\Omega)\right)^{3}}=-\left(\frac{1}{\mu_{\alpha}} \operatorname{curl} u_{g}, \operatorname{curl} v_{h}\right)_{\left(L^{2}(\Omega)\right)^{3}} \quad \forall v_{h} \in \mathcal{H}_{h}, \\
\left(\varepsilon_{\alpha} \mathcal{E}_{h}, \operatorname{grad} \psi_{h}\right)_{\left(L^{2}(\Omega)\right)^{3}}=-\left(\varepsilon_{\alpha} u_{g}, \operatorname{grad} \psi_{h}\right)_{\left(L^{2}(\Omega)\right)^{3}} \quad \forall \psi_{h} \in \Psi_{h} .
\end{array}\right.
$$

According to properties of the present finite elements, as well to the definitions of $\mu_{\alpha}, \varepsilon_{\alpha}$ and to the assumptions on the geometry of $\Omega$, it follows that any solution of the discrete formulation (4.3) is unique. As mentioned earlier, the numerical approximation of $\mathcal{E}_{\alpha}$ does not require the discretization of the potential $q$. We will be concerned with the formulation (4.3) in numerical simulations since it allows us to approximate the electric field $E_{\alpha}$, through (3.6), by considering the vector field

$$
E_{\alpha}^{h}:=\mathcal{E}_{h}+u_{g}
$$

as the discrete electric field. In fact, (4.3) represents the discrete problem associated with the continuous problem (3.5) satisfied by $\mathcal{E}_{\alpha}$.

For the evaluation of the integral terms of the formulation (4.3), we use a numerical integration method of order 2. A rectangular linear system results from this formulation and we are concerned in computations with the determination of a least squares solution. More precisely, we make use of the normal equations associated with the system, and apply a (complex) conjugate gradient-type method (see, e.g., [14]) for solving these equations. Since such equations are considered, preconditioning of the minimization method is required and we use a preconditioner that is an upper banded matrix, corresponding to the triangular matrix in an Incomplete Modified Gram-Schmidt (IMGS) factorization of the matrix of the system — with a row major ordering (see [23] for an analysis of this preconditioning technique).

\section{Localization Procedures}

In this part, we describe two procedures for the localization of the imperfections. Each procedure results from the combination of the formula (3.10) with one of the following inversion algorithms: the Current Projection method or an Inverse Fourier method. 


\subsection{Procedure based on the Current Projection method}

This is a localization method which can be used only in the case where the domain contains a single imperfection. Our aim in this case is to determine the center of the imperfection. Let us first describe how we make use of the formula (3.10). If we denote by $p=\left(p_{1}, p_{2}, p_{3}\right)^{T}$ the center of the imperfection, by $M$ the "rescaled" polarization tensor $\left(\frac{\mu_{0}}{\mu_{1}}-1\right) M^{1}\left(\frac{\mu_{0}}{\mu_{1}}\right)$ of this imperfection, it follows from (3.10) that:

$$
\begin{aligned}
\Gamma & :=\int_{\partial \Omega} \operatorname{curl} E_{\alpha} \times \nu \cdot w d \sigma-\int_{\partial \Omega} \operatorname{curl} w \times \nu \cdot(\nu \times g) d \sigma \\
& \approx \alpha^{3}\left(M \operatorname{curl} E_{0}(p)\right) \cdot \operatorname{curl} w(p)
\end{aligned}
$$

with $g=E_{\alpha} \times \nu$, and $w$ any smooth vector-valued function satisfying (3.11).

Let us recall that following (3.6), we have $E_{\alpha}=\mathcal{E}_{\alpha}+u_{g}$, where $u_{g}$ is introduced in (3.4) and $\mathcal{E}_{\alpha}$ verifies (3.5). The datum $g$, that also defines $u_{g}$, is considered from a physical point of view as a current applied on $\partial \Omega$. The discrete field $\mathcal{E}_{h}$ associated with $\mathcal{E}_{\alpha}$ is the least squares solution of the discrete formulation (4.3), and the discrete electric field associated with $E_{\alpha}$ is defined as: $E_{\alpha}^{h}:=\mathcal{E}_{h}+u_{g}$.

Let us apply different currents for $g$ that correspond to the following background vector potentials:

$$
E_{0}^{(1)}\left(x_{1}, x_{2}, x_{3}\right)=\left(\begin{array}{c}
0 \\
0 \\
x_{2}
\end{array}\right), E_{0}^{(2)}\left(x_{1}, x_{2}, x_{3}\right)=\left(\begin{array}{c}
x_{3} \\
0 \\
0
\end{array}\right), E_{0}^{(3)}\left(x_{1}, x_{2}, x_{3}\right)=\left(\begin{array}{c}
0 \\
x_{1} \\
0
\end{array}\right) .
$$

For each current $g^{(i)}=E_{0}^{(i)} \times \nu, 1 \leq i \leq 3$, we put $g:=g^{(i)}$ in (3.4) and compute by (4.3) the corresponding discrete electric field denoted here by $E_{\alpha,(i)}^{h}$. Next, we consider the test vector fields

$$
w^{(1)}\left(x_{1}, x_{2}, x_{3}\right)=\left(\begin{array}{c}
0 \\
0 \\
x_{2}
\end{array}\right), w^{(2)}\left(x_{1}, x_{2}, x_{3}\right)=\left(\begin{array}{c}
x_{3} \\
0 \\
0
\end{array}\right), w^{(3)}\left(x_{1}, x_{2}, x_{3}\right)=\left(\begin{array}{c}
0 \\
x_{1} \\
0
\end{array}\right),
$$

in order to evaluate from the left-hand side of (5.1) the terms $\Gamma_{(j, i)}, 1 \leq j \leq 3$, defined as:

$$
\Gamma_{(j, i)}:=\int_{\partial \Omega} \operatorname{curl} E_{\alpha,(i)}^{h} \times \nu \cdot w^{(j)} d \sigma-\int_{\partial \Omega} \operatorname{curl} w^{(j)} \times \nu \cdot\left(\nu \times g^{(i)}\right) d \sigma .
$$

Each $\Gamma_{(j, i)}, 1 \leq i, j \leq 3$, is called the measurement, and its evaluation is achieved with the aid of a numerical integration method of order 2. By using the formula (5.1), it follows from (5.2) that:

$$
\Gamma_{(j, i)} \approx \alpha^{3} M_{j i}
$$

where the terms $M_{j i}, 1 \leq i, j \leq 3$, are the coefficients of $M$. We obtain then in this way an approximation of the rescaled tensor $\alpha^{3} M$. 
Once an approximation of this tensor is determined, we can localize the center of the imperfection by using the same background vector potentials and by now considering

$$
w^{(4)}\left(x_{1}, x_{2}, x_{3}\right)=\left(\begin{array}{c}
x_{2} x_{3} \\
-x_{1} x_{3} \\
0
\end{array}\right)
$$

as another test vector field. In fact, we compute now, for $1 \leq i \leq 3$, the measurement

$$
\Gamma_{(4, i)}:=\int_{\partial \Omega} \operatorname{curl} E_{\alpha,(i)}^{h} \times \nu \cdot w^{(4)} d \sigma-\int_{\partial \Omega} \operatorname{curl} w^{(4)} \times \nu \cdot\left(\nu \times g^{(i)}\right) d \sigma,
$$

and build from (5.1) the following "linear system":

$$
\left\{\begin{array}{l}
\Gamma_{(4,1)} \approx \alpha^{3} M_{11} p_{1}+\alpha^{3} M_{21} p_{2}-2 \alpha^{3} M_{31} p_{3}, \\
\Gamma_{(4,2)} \approx \alpha^{3} M_{12} p_{1}+\alpha^{3} M_{22} p_{2}-2 \alpha^{3} M_{32} p_{3}, \\
\Gamma_{(4,3)} \approx \alpha^{3} M_{13} p_{1}+\alpha^{3} M_{23} p_{2}-2 \alpha^{3} M_{33} p_{3},
\end{array}\right.
$$

which allows us to determine in a unique way the unknown $\left(p_{1}, p_{2}, p_{3}\right)^{T}$. This will always be possible while $\mu_{1} \neq \mu_{0}$ and when the polarization tensor $M^{1}\left(\frac{\mu_{0}}{\mu_{1}}\right)$ is positive definite, namely when $\mu_{0}>0$ and $\mu_{1}>0$. Of course, in this context, the matrix of the "system" (5.4) is invertible since its determinant is equal to $-2 \operatorname{det}\left(\alpha^{3} M\right)$ and $\alpha^{3} M$ is invertible.

By considering therefore three background vector potentials, and also four test vector fields, we determine both an approximation of the tensor $\alpha^{3} M$ when $\mu_{1} \neq \mu_{0}$ and an approximation of the center of the imperfection, for of course $\mu_{0}>0, \mu_{1}>0$.

The measurements in (5.3) do not report any information in the purely electric case, namely when $\mu_{1}=\mu_{0}$ and $\varepsilon_{0}>0, \varepsilon_{1}>0, \varepsilon_{1} \neq \varepsilon_{0}$.

When the rescaled polarization tensor $\left(\frac{\mu_{0}}{\mu_{1}}-1\right) M^{1}\left(\frac{\mu_{0}}{\mu_{1}}\right)$ is known, an approximation of the order of magnitude of the diameter of the imperfection can be obtained from one of the measurements $\Gamma_{(i, i)}, 1 \leq i \leq 3$, while $\mu_{1} \neq \mu_{0}$, and of course $\mu_{0}>0, \mu_{1}>0$.

\subsection{Procedure based on an Inverse Fourier method}

We are here interested in a variational method based on the original idea of Calderón [9] which was to reduce the localization problem to the calculation of an inverse Fourier transform.

First of all, let us reconsider the formula (3.10) as follows

$$
\begin{aligned}
& \Gamma:=\int_{\partial \Omega} \operatorname{curl} E_{\alpha} \times \nu \cdot w d \sigma-\int_{\partial \Omega} \operatorname{curl} w \times \nu \cdot(\nu \times g) d \sigma \\
& \approx \alpha^{3} \sum_{j=1}^{m}\left(\frac{\mu_{0}}{\mu_{j}}-1\right)\left[M^{j}\left(\frac{\mu_{0}}{\mu_{j}}\right) \operatorname{curl} E_{0}\left(z_{j}\right)\right] \cdot \operatorname{curl} w\left(z_{j}\right),
\end{aligned}
$$

where $g=E_{\alpha} \times \nu$. For an arbitrary $\eta \in \mathbb{R}^{3}$, let us define $\beta$ and $\zeta$ in $\mathbb{R}^{3}$ such that:

$$
\left\{\begin{array}{l}
\|\beta\|^{2}=1, \quad \beta \cdot \eta=0 \\
\|\zeta\|^{2}=1, \quad \zeta \cdot \eta=\zeta \cdot \beta=0
\end{array}\right.
$$


with $\|\cdot\|$ denoting the usual norm associated with the Hermitian product on $\mathbb{C}^{3}$. Let $p=\eta+\gamma \beta$, where $\gamma$ is a complex number such that $\gamma=\mathrm{i}\|\eta\|$, i.e., $p \cdot p=0$. We assume that we are in possession of the boundary current for the electric field $E_{\alpha}$, whose corresponding background potential is given by

$$
E_{0}(x)=\mathrm{e}^{\mathrm{i} p \cdot x} \zeta
$$

In fact, in (5.5), we set $g(x)=\left(\mathrm{e}^{\mathrm{i} p \cdot x} \zeta\right) \times \nu(x)$ and use as the test vector field, $w(x)=\mathrm{e}^{\mathrm{i} q \cdot x} \zeta$, where $q=\eta-\gamma \beta$. Namely, as well as $E_{0}$, the vector field $w$ is in accordance with (3.11). With these considerations of $g$ and $w$, we get from (5.5) that

$$
\Gamma \approx \alpha^{3} \sum_{j=1}^{m}\left(\left(\frac{\mu_{0}}{\mu_{j}}-1\right)\left[M^{j}\left(\frac{\mu_{0}}{\mu_{j}}\right)\left(\mathrm{ie}^{\mathrm{i} p \cdot z_{j}} p \times \zeta\right)\right] \cdot\left(\mathrm{ie}^{\mathrm{i} q \cdot z_{j}} q \times \zeta\right)\right) .
$$

Let us now view the measurement as a function of $\eta$ :

$$
\Gamma(\eta) \approx \alpha^{3} \sum_{j=1}^{m}\left(-\left(\frac{\mu_{0}}{\mu_{j}}-1\right)\left[M^{j}\left(\frac{\mu_{0}}{\mu_{j}}\right)((\eta+\gamma \beta) \times \zeta)\right] \cdot((\eta-\gamma \beta) \times \zeta)\right) \mathrm{e}^{\mathrm{i} 2 \eta \cdot z_{j}}
$$

By inspecting the context where all the imperfections are balls - the tensors $M^{j}(c)$ being accordingly of the form $m^{j}(c) \mathrm{I}_{3}$, with $\mathrm{I}_{3}$ the $3 \times 3$ identity matrix, $m^{j}(c)$ a scalar depending on $c$ (see, e.g., [7]), it follows that

$$
\Gamma(\eta) \approx \alpha^{3} \sum_{j=1}^{m}\left[-\left(\frac{\mu_{0}}{\mu_{j}}-1\right) m^{j}\left(\frac{\mu_{0}}{\mu_{j}}\right)\left(2\|\eta\|^{2}\right)\right] \mathrm{e}^{\mathrm{i} 2 \eta \cdot z_{j}}
$$

Recall that the function $\mathrm{e}^{\mathrm{i} 2 \eta \cdot z_{j}}$ (up to a multiplicative constant) is exactly the Fourier transform of the Dirac delta $\delta_{-2 z_{j}}$ (a point mass located at $-2 z_{j}$ ). Since $\|\eta\|^{2}$ is a polynomial in the coordinates $\eta_{i}, 1 \leq i \leq 3$, of $\eta$, we have in the right-hand side of (5.7) multiplications by powers of $\eta_{i}$ in Fourier space that correspond to differentiations of the delta functions. In this particular context, the expression in the right-hand side of (5.7) is therefore the Fourier transform of a linear combination of derivatives of order less than or equal to 2 of delta functions centered at the points $-2 z_{j}, 1 \leq j \leq m$. More precisely, the inverse Fourier transform of $\Gamma(\eta)$ is expressed as:

$$
\check{\Gamma}(x) \approx \alpha^{3} \sum_{j=1}^{m} L_{j}\left(\delta_{-2 z_{j}}\right)(x)
$$

where $L_{j}$ is a second-order differential operator with constant coefficients depending on $m^{j}\left(\frac{\mu_{0}}{\mu_{j}}\right)$. In this approach, a numerical Fourier inversion of a sample of measurements should efficiently pin down the $z_{j}$ 's. The inversion principle that will be described here is similar to the one presented by Volkov in [22] in the context of a localization of imperfections from an inverse problem based on the two-dimensional Helmholtz equation. This principle has been also successfully used (see [7]) in the context of the localization of three-dimensional electromagnetic imperfections from the model (2.6).

When some of the imperfections are not balls, we may rewrite (5.6) as below, where the measurement $\Gamma$ is viewed again as a function of $\eta$ :

$$
\Gamma(\eta) \approx \alpha^{3} \sum_{j=1}^{m}\left(-\left(\frac{\mu_{0}}{\mu_{j}}-1\right) T_{\mu_{0}, \mu_{j}}(\eta)\right) \mathrm{e}^{\mathrm{i} 2 \eta \cdot z_{j}}
$$


with $T_{\mu_{0}, \mu_{j}}(\eta)=\left(M^{j}\left(\frac{\mu_{0}}{\mu_{j}}\right)((\eta+\gamma \beta) \times \zeta)\right) \cdot((\eta-\gamma \beta) \times \zeta)$. The expression in the right-hand side of (5.8) is in fact the Fourier transform of an operator of a more complicated kind acting on delta functions centered at the points $-2 z_{j}, 1 \leq j \leq m$. The present inversion principle consists of sampling $\Gamma(\eta)$ at some discrete set of points and then evaluating the discrete inverse Fourier transform of the corresponding sample. After a rescaling (by $-\frac{1}{2}$ ), the support of this inverse Fourier transform will provide the locations of the imperfections.

Typically, for each point $\eta$ (of the mentioned discrete set), we consider $g(x)=\left(\mathrm{e}^{\mathrm{i}(\eta+\gamma \beta) \cdot x} \zeta\right) \times$ $\nu(x)$ as the boundary current in (3.4) and compute through (4.3) the corresponding discrete electric field, denoted here by $E_{\alpha}^{h}$. After determining the discrete field, $\operatorname{curl} E_{\alpha}^{h} \times \nu$, we evaluate numerically, with the aid of an integration formula of order 2 , the measurement $\Gamma(\eta)$ by using of course $w(x)=\mathrm{e}^{\mathrm{i}(\eta-\gamma \beta) \cdot x} \zeta$ as the test field in $\int_{\partial \Omega} \operatorname{curl} E_{\alpha} \times \nu \cdot w d \sigma-\int_{\partial \Omega} \operatorname{curl} w \times \nu \cdot(\nu \times g) d \sigma$, and by replacing $E_{\alpha}$ by $E_{\alpha}^{h}$ in this difference of terms representing in fact the left-hand side of (5.6).

Let us now specify, following [22], a possible way to choose a step size for sampling with respect to $\eta$ in the numerical simulations.

First of all, let us assume that all the centers $z_{j}=\left(z_{j}^{1}, z_{j}^{2}, z_{j}^{3}\right)^{T}$ of the imperfections $(1 \leq$ $j \leq m$ ) lie in a domain $[-K, K]^{3}$, where the bound $K$ is known. To simplify the presentation, let us consider, for instance, the formula (5.7) and rewrite simply its right-hand side as:

$$
\sum_{j=1}^{m} C_{j} \mathrm{e}^{2 \mathrm{i}\left(\eta_{1} z_{j}^{1}+\eta_{2} z_{j}^{2}+\eta_{3} z_{j}^{3}\right)}
$$

where the complex constants $C_{j}$ are unknown. As previously mentioned, for each $\eta=\left(\eta_{1}, \eta_{2}, \eta_{3}\right)^{T}$, we are able to evaluate the measurement $\Gamma(\eta)$ and therefore we assume that $(5.9)$ is known for $\left(\eta_{1}, \eta_{2}, \eta_{3}\right)^{T} \in\left[-\eta_{\max }, \eta_{\max }\right]^{3}$, on a regular grid made up of $n^{3}$ points. We are then in possession of the sequence of data:

$$
\sum_{j=1}^{m} C_{j} \mathrm{e}^{2 \mathrm{i}\left(\left(-\eta_{\max }+\left(l_{1}-1\right) \rho\right) z_{j}^{1}+\left(-\eta_{\max }+\left(l_{2}-1\right) \rho\right) z_{j}^{2}+\left(-\eta_{\max }+\left(l_{3}-1\right) \rho\right) z_{j}^{3}\right)}, \quad 1 \leq l_{1}, l_{2}, l_{3} \leq n,
$$

where $\rho=2 \eta_{\max } / n$. After applying the inverse Fourier transform to this sequence, we get

$$
\begin{array}{r}
\frac{1}{n^{3}} \sum_{j=1}^{m} C_{j} \sum_{1 \leq l_{1}, l_{2}, l_{3} \leq n} \mathrm{e}^{2 \mathrm{i}\left(\left(-\eta_{\max }+\left(l_{1}-1\right) \rho\right) z_{j}^{1}+\left(-\eta_{\max }+\left(l_{2}-1\right) \rho\right) z_{j}^{2}+\left(-\eta_{\max }+\left(l_{3}-1\right) \rho\right) z_{j}^{3}\right)} \\
\times \mathrm{e}^{2 \mathrm{i} \pi\left(\frac{\left(l_{1}-1\right)}{n}\left(s_{1}-1\right)+\frac{\left(l_{2}-1\right)}{n}\left(s_{2}-1\right)+\frac{\left(l_{3}-1\right)}{n}\left(s_{3}-1\right)\right),},
\end{array}
$$

with $1 \leq s_{1}, s_{2}, s_{3} \leq n$. Let us now reduce the module of the term in (5.10) as follows:

$$
\left|\sum_{j=1}^{m} \frac{8 C_{j}}{n^{3}} \frac{\sin \left(2 \eta_{\max } z_{j}^{1}\right) \sin \left(2 \eta_{\max } z_{j}^{2}\right) \sin \left(2 \eta_{\max } z_{j}^{3}\right)}{\left(\mathrm{e}^{2 \pi\left(\frac{\rho z_{j}^{1}}{\pi}+\frac{s_{1}-1}{n}\right) \mathrm{i}}-1\right)\left(\mathrm{e}^{2 \pi\left(\frac{\rho z_{j}^{2}}{\pi}+\frac{s_{2}-1}{n}\right) \mathrm{i}}-1\right)\left(\mathrm{e}^{2 \pi\left(\frac{\rho z_{j}^{3}}{\pi}+\frac{s_{3}-1}{n}\right) \mathrm{i}}-1\right)}\right| .
$$

Then, as $n$ becomes large, the quantity in (5.11) is small unless one of the terms $\rho z_{j}^{1} / \pi+\left(s_{1}-\right.$ $1) / n, \quad \rho z_{j}^{2} / \pi+\left(s_{2}-1\right) / n$ and $\rho z_{j}^{3} / \pi+\left(s_{3}-1\right) / n$ is close to an integer. By enforcing (for example)

$$
\frac{K \rho}{\pi} \lesssim \frac{1}{3}
$$


each one of the previous terms shall only approach the integers 0 or 1 , in the case where $n$ becomes large $(n \geq 3)$. The relation $(5.12)$ provides a practical way to choose the step size $\rho$ and also indicates a link among $\eta_{\max }, K$ and $n$. In fact, we have $\rho=2 \eta_{\max } / n$ and take from $(5.12)$

$$
\rho \approx \frac{1}{K}
$$

In this approach, we shall fix $\rho$ according to (5.13) and consider simultaneously increasing values of $n$ and of $\eta_{\max }$ for more accuracy. This is a localization procedure whose centers $z_{j}$, $1 \leq j \leq m$, are localized from the sequence of the modules of the terms in (5.10), with at best (theoretically) a resolution of order $\pi /\left(2 \eta_{\max }\right)$ (see also [7]), and after a rescaling (by $\left.-\frac{1}{2}\right)$. This procedure provides of course a sampling of the "physical" domain in association with the considered sampling for $\left[-\eta_{\max }, \eta_{\max }\right]^{3}$.

\section{Numerical Simulations}

We present here, in various contexts, the numerical results of the effective localization of the imperfections, obtained by using the procedures previously introduced.

\subsection{Computational configurations}

Two distinct configurations of the (polyhedral) domain $\Omega$, having here the diameter and the shape of the unit ball, are considered: the case where $\Omega$ contains a single imperfection and when it contains multiple imperfections. For the first configuration, the single imperfection is a polyhedron having the shape of a ball of center $p=\left(p_{1}, p_{2}, p_{3}\right)^{T} \in \Omega$ and of radius $\alpha$. We represent the discretization of $\bar{\Omega}$ by

- $\mathcal{T}_{\alpha}^{1}$ when $p=(0.23,-0.31,0.15)^{T}$, with $\alpha=0.2$;

- $\mathcal{T}_{\alpha}^{2}$ when $p=(0.23,-0.31,0.15)^{T}$, with $\alpha=0.17$.

In the second configuration, each imperfection is a polyhedron having the shape of a ball or of an ellipsoid. The discretization of $\bar{\Omega}$ is now represented by

- $\mathcal{T}_{\alpha}^{3}$ when $\Omega$ contains two ball-like shaped imperfections of centers $(0.23,-0.31,0.15)^{T}$, $(-0.17,0.43,-0.11)^{T}$, and of the same 'radius' $\alpha=0.2$;

- $\mathcal{T}_{\alpha}^{4}$ when $\Omega$ contains three ball-like shaped imperfections of centers $(0.23,-0.31,0.15)^{T}$, $(-0.17,-0.43,-0.11)^{T},(-0.5,0.25,0.1)^{T}$, with respective 'radii' $0.18,0.16$ and 0.17 . In this case, we denote by $\alpha$ the maximal radius and by $\alpha_{\min }$ the minimal radius: $\alpha=0.18$, $\alpha_{\min }=0.16$;

- $\mathcal{T}_{\alpha}^{5}$ when $\Omega$ contains three imperfections one of which has the shape of a ball of radius 0.16 and of center $(0.23,-0.31,0.15)^{T}$. The second one has the shape of an ellipsoid centered at $(-0.17,-0.43,-0.11)^{T}$ with semi-axes of lengths $0.16,0.16,0.18$ in the directions $O x, O y, O z$ respectively. The last imperfection is also ellipsoid-shaped, but centered at $(-0.5,0.25,0.1)^{T}$ with the 'semi-axes' (on $O x y$ ) rotated about $O z$ by an angle of $\pi / 4$. The lengths of the 'semi-axes' of this imperfection are $0.16,0.17$ and 0.19 . Now, $\alpha$ is the maximal value of the semi-axes lengths and the 'radius' of the first imperfection, and $\alpha_{\min }$ is the minimal value of these quantities: $\alpha=0.19, \alpha_{\min }=0.16$. 
Of course, each one of these discretizations is associated with a conforming mesh made up of tetrahedra that takes implicitly into account the geometry of each imperfection. The resulting mesh size $h$ is here systematically smaller than the lowest of the 'radii' or 'semi-axes lengths' of the imperfections: $h<\alpha_{\min }$. In the following table, we give some characteristics of the mesh in each one of these settings.

Table 6.1 The characteristics of the mesh.

\begin{tabular}{|c|c|c|c|c|c|c|}
\hline & $N K$ & NIE & NIV & $n f$ & $n e$ & $h$ \\
\hline $\mathcal{T}_{\alpha}^{1}$ & 45101 & 49906 & 6643 & 3678 & 5517 & 0.17725 \\
\hline $\mathcal{T}_{\alpha}^{2}$ & 54368 & 60753 & 8215 & 3662 & 5493 & 0.15717 \\
\hline $\mathcal{T}_{\alpha}^{3}$ & 55847 & 62386 & 8425 & 3774 & 5661 & 0.15718 \\
\hline $\mathcal{T}_{\alpha}^{4}$ & 64765 & 72662 & 9872 & 3952 & 5928 & 0.14810 \\
\hline $\mathcal{T}_{\alpha}^{5}$ & 74093 & 83334 & 11363 & 4246 & 6369 & 0.14534 \\
\hline
\end{tabular}

We have denoted by $N K, N I E, N I V$ the number of tetrahedra, internal edges and internal vertices respectively. Also, $n f$, ne are respectively the number of boundary faces and boundary edges.

Let us mention that the settings considered above were also used (see [7]) in the context of the localization of the same imperfections from the model presented in (2.6).

\subsection{Using the procedure based on the Current Projection method}

By making use of the procedure based on the Current Projection method (see Subsection 5.1 , we describe here the results of the numerical localization of a single imperfection. Typically, we consider $(5.3)-(5.4)$ by distinguishing the cases: $\mu_{1} \neq \mu_{0}$ with $\varepsilon_{1}=\varepsilon_{0}$, and $\mu_{1} \neq \mu_{0}$ with $\varepsilon_{1} \neq \varepsilon_{0}$, after fixing $\mu_{0}=\varepsilon_{0}=1$. Of course, the purely electric case is here excluded in conformity with Remark 3.3.

As specified in Subsection 5.1, the reconstruction of the center of the imperfection will always be possible from (5.4) while the measurements in (5.3) and (5.4) are known, namely for $\mu_{1} \neq \mu_{0}, \mu_{1}>0$. Regarding the order of magnitude of the diameter of the imperfection, and more precisely, here, the reconstruction of the 'radius' $\alpha$, our simulations require of course the numerical evaluation of the polarization tensor $M^{1}\left(\frac{\mu_{0}}{\mu_{1}}\right)$ corresponding to the imperfection. This evaluation is done as explained (in another context of investigations) in [7], by calculating numerically the coefficients of the tensor from (2.5), with $B_{j_{0}} \equiv B_{1}$ identified then with a polyhedral domain having the shape and the diameter of the unit ball, and after discretizing the solution of (2.4) from a combination of interior nodal finite elements with boundary finite elements of first-order (see also e.g. [17] for such a combination). As mentioned earlier, the reconstruction of the 'radius' $\alpha$ will be performed while the measurements in (5.3) are moreover evaluated.

Let us respectively represent by $\left|\alpha-\alpha_{h}\right| /|\alpha|$ and $\left|p-p_{h}\right|_{\mathbf{R}^{3}} /|p|_{\mathbf{R}^{3}}$ (with $|\cdot|_{\mathbf{R}^{3}}$ denoting the infinity norm on $\mathbb{R}^{3}$ ), the relative errors on the 'radius' $\alpha$ and the center $p$ of the imperfection, when $\alpha_{h}, p_{h}$ are the 'radius' and the center of the localized imperfection.

Figs. 6.1-6.2 present the results obtained from the reconstruction of the 'radius' $\alpha$, with the settings $\mathcal{T}_{\alpha}^{1}$ and $\mathcal{T}_{\alpha}^{2}$, and for $\varepsilon_{1}=1,5,10,100$. We observe an asymptotic behavior of the relative error on the radius of the imperfection with respect to $\mu_{1}\left(\mu_{1}>\mu_{0}\right)$. We also notice 

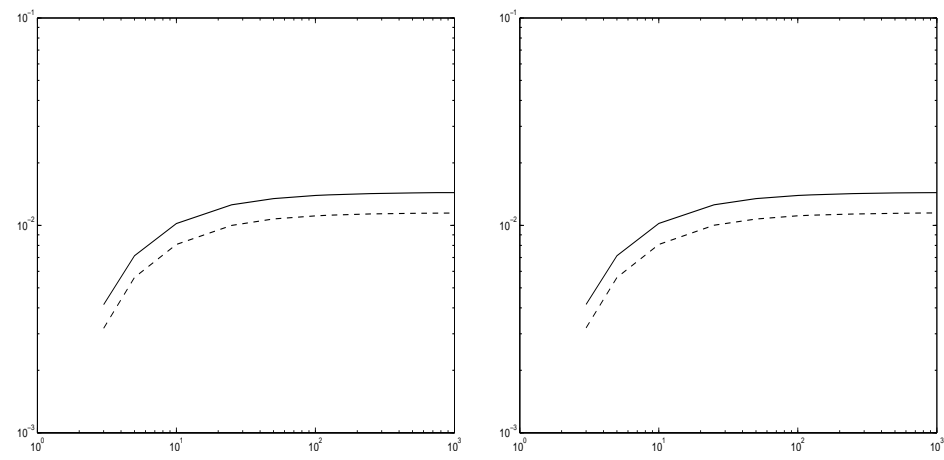

Fig. 6.1. Log-log representation of the relative error on the radius with respect to some values of $\mu_{1}$, with $\varepsilon_{1}=1$ (at left) and $\varepsilon_{1}=5$ (at right), from the settings $\mathcal{T}_{\alpha}^{1}(-)$ and $\mathcal{T}_{\alpha}^{2}(---)$.
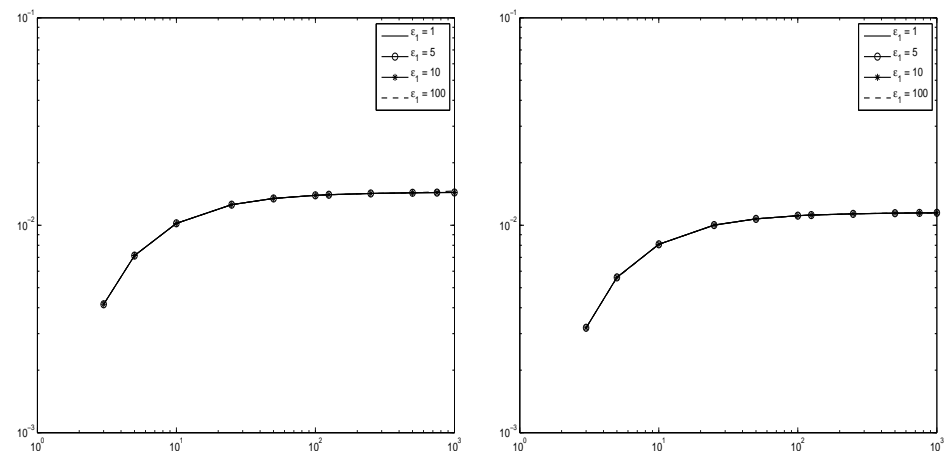

Fig. 6.2. Log-log representation of the relative error on the radius with respect to some values of $\mu_{1}$, and with $\varepsilon_{1}=1,5,10,100$, from the settings $\mathcal{T}_{\alpha}^{1}$ (at left) and $\mathcal{T}_{\alpha}^{2}$ (at right).
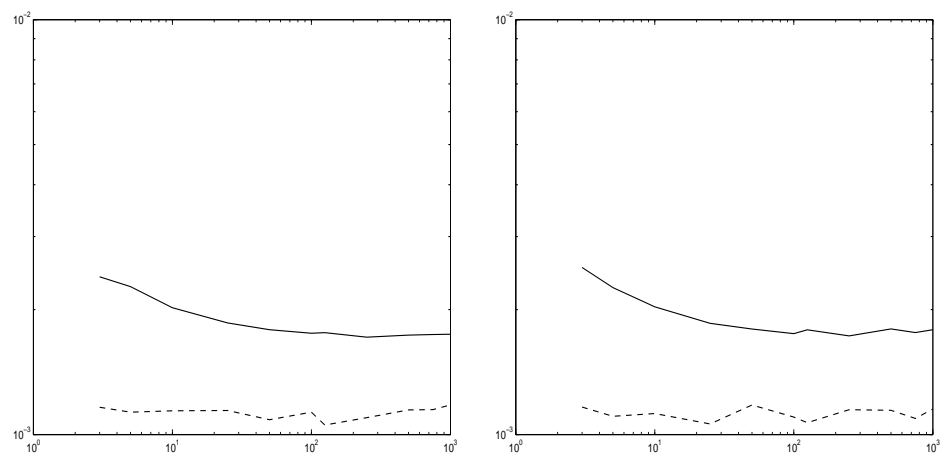

Fig. 6.3. Log-log representation of the relative error on the center with respect to some values of $\mu_{1}$, with $\varepsilon_{1}=1$ (at left) and $\varepsilon_{1}=5$ (at right), from the settings $\mathcal{T}_{\alpha}^{1}(-)$ and $\mathcal{T}_{\alpha}^{2}(---)$.

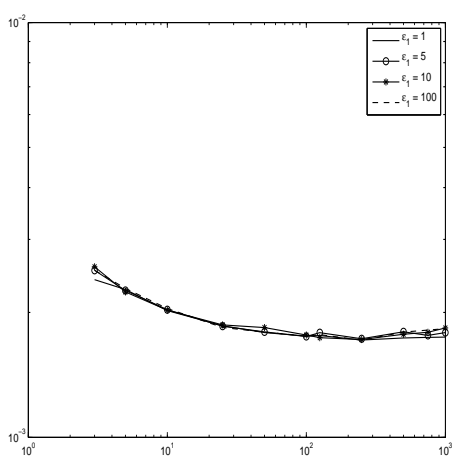

Fig. 6.4. Log-log representation of the relative error on the center with respect to some values of $\mu_{1}$, with $\varepsilon_{1}=$ $1,5,10,100$, from the setting $\mathcal{T}_{\alpha}^{1}$.

that the relative error on the radius obtained from $\mathcal{T}_{\alpha}^{2}$ is asymptotically slightly smaller than the one resulting from $\mathcal{T}_{\alpha}^{1}$. Independently of the considered setting, the relative error on the radius increases with respect to $\mu_{1}$.

Fig. 6.2 also indicates that this relative error does not vary significantly with respect to $\varepsilon_{1}$, 

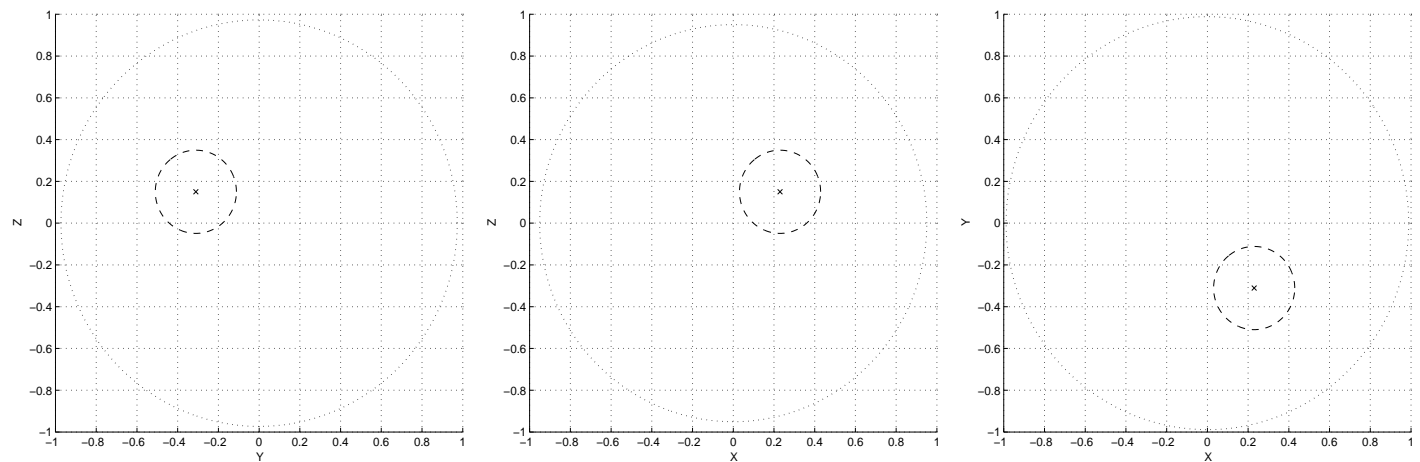

Fig. 6.5. Respective cross-sections at $x=p_{1}, y=p_{2}$ and $z=p_{3}$, of the localized imperfection $(---)$ with its center marked by " $\times$ ", from the setting $\mathcal{T}_{\alpha}^{1}$ and with $\mu_{1}=3, \varepsilon_{1}=1$.
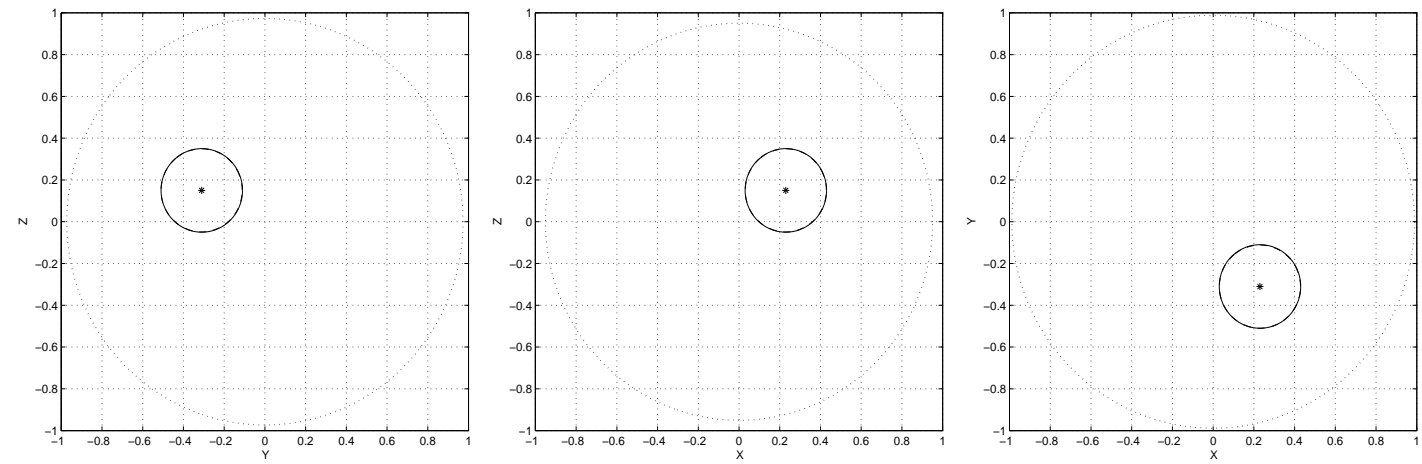

Fig. 6.6. Respective cross-sections at $x=p_{1}, y=p_{2}$ and $z=p_{3}$, from the setting $\mathcal{T}_{\alpha}^{1}$ and with $\mu_{1}=3$, $\varepsilon_{1}=1$. Superposition of the original imperfection (-) whose center is marked by "+", and of the localized imperfection $(---)$ with its center marked by " $\times$ ".

for each fixed value of $\mu_{1}$ and each considered setting.

Figs. 6.3-6.4 concern the results of the reconstruction of the center of the imperfection, also obtained from the settings $\mathcal{T}_{\alpha}^{1}$ and $\mathcal{T}_{\alpha}^{2}$, with $\varepsilon_{1}=1,5,10,100$. The relative error on the center obtained from $\mathcal{T}_{\alpha}^{2}$ is slightly smaller than the one resulting from $\mathcal{T}_{\alpha}^{1}$. Since a similar smallness has been noticed for the relative error on the radius, we can hence expect with $\mathcal{T}_{\alpha}^{2}$ better reconstructions of the imperfection than with $\mathcal{T}_{\alpha}^{1}$.

As indicated by Fig. 6.4, the relative error on the center does not vary significantly with respect to $\varepsilon_{1}$, for each fixed value of $\mu_{1}$ and for the considered setting.

On the other hand, the variation of this relative error is not asymptotically large with respect to $\mu_{1}$, for each considered setting.

Fig. 6.5 shows the cross-sections at $x=p_{1}=0.23, y=p_{2}=-0.31$ and $z=p_{3}=0.15$, of the localized imperfection resulting from $\mathcal{T}_{\alpha}^{1}$ when $\mu_{1}=3, \varepsilon_{1}=1$.

When we superpose, as in Fig. 6.6, the cross-sections at $x=p_{1}, y=p_{2}$ and $z=p_{3}$, of the original imperfection (with center $\left(p_{1}, p_{2}, p_{3}\right)^{T}$ ) and of this localized imperfection, there is not a significant difference between the representations. This comes from the fact that the related relative errors on the radius and the center are very small. For this reason, we will represent below only the cross-sections of the localized imperfection, with respect to each considered physical contrast.

Fig. 6.7 shows the cross-sections again in the context of $\mathcal{T}_{\alpha}^{1}$ but in an electromagnetic case. 

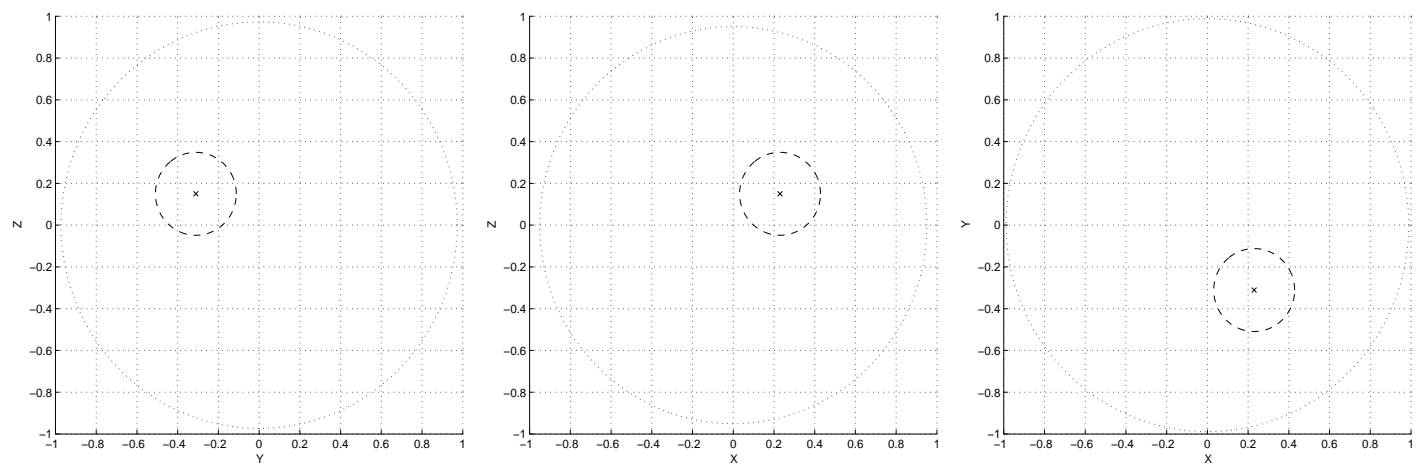

Fig. 6.7. Same as Fig. 6.5 except with $\mu_{1}=5, \varepsilon_{1}=10$.
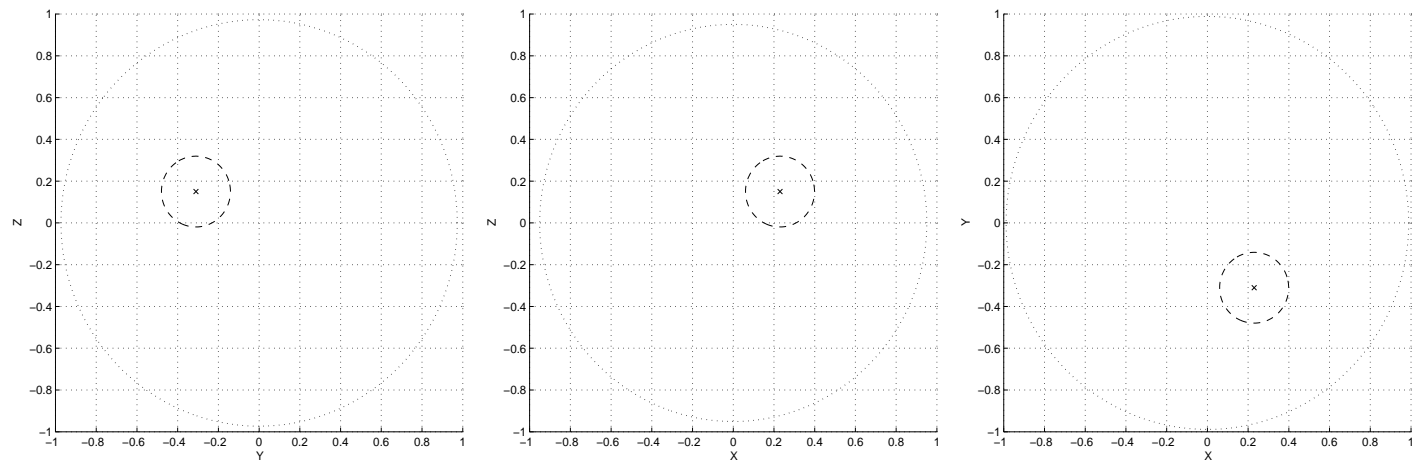

Fig. 6.8. Respective cross-sections at $x=p_{1}, y=p_{2}$ and $z=p_{3}$, of the localized imperfection (- - - ) with its center marked by " $\times$ ", from the setting $\mathcal{T}_{\alpha}^{2}$ and with $\mu_{1}=3, \varepsilon_{1}=1$.
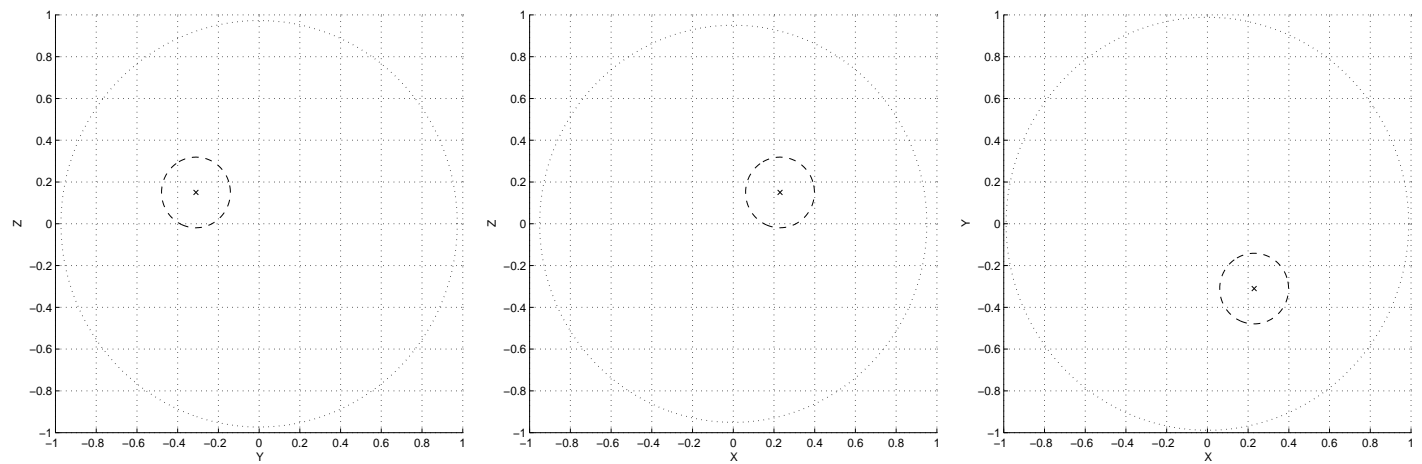

Fig. 6.9. Same as Fig. 6.8 except with $\mu_{1}=5, \varepsilon_{1}=3$.

The results represented in Figs. 6.8-6.9 concern the localization of an inhomogeneity shaped and centered as the one defined from $\mathcal{T}_{\alpha}^{1}$, but having a smaller size. The experiment associated with Fig. 6.8 is done in a magnetic context whereas an electromagnetic contrast is taken into account in the experiment related to Fig. 6.9.

The fact that reconstructions of the imperfection defined from $\mathcal{T}_{\alpha}^{2}$ are better suggests that the formula (3.10) is numerically more accurate when we use $\mathcal{T}_{\alpha}^{2}$ which considers a smaller imperfection than the one defined from $\mathcal{T}_{\alpha}^{1}$. 
Similar localizations to those represented in Figs. 6.5-6.9 are obtained from simulations based on $\mathcal{T}_{\alpha}^{1}, \mathcal{T}_{\alpha}^{2}$, when we consider other values of $\mu_{1}$ and $\varepsilon_{1}$ as for example the ones used in Fig. 6.2. Let us also mention that the evaluation with good numerical accuracy of the measurements required in (5.2) and (5.4) has contributed to obtain very accurate results in this subsection.

\subsection{Using the procedure based on an Inverse Fourier method}

The procedure based on an Inverse Fourier method is considered here to perform numerical simulations in each one of the configurations where $m=1$ or $m>1$. The "illumination" of the domain $\Omega$ with incident waves is applied in conformity with the sampling in the Fourier space that provides a discrete Fourier domain, constituted of $n^{3}$ points and encapsulated by $\left[-\eta_{\max }, \eta_{\max }\right]^{3}$. Recall that each incident wave is associated with a boundary measurement numerically evaluated through finite element computations, and in total $n^{3}$ sampled measurements define in fact the data of the procedure. The outcome of the procedure is the sequence of modules of the terms that approximate those of (5.10), following the formula for measurements. The presentation of our results will consist here of representing, after a rescaling by $-1 / 2$, contour-plots based on this sequence, additionally enriched by a usual linear interpolation process.

Although large values of $\eta_{\max }$ should be considered in the simulations in order to expect accurate localizations (following Subsection 5.2), a direct application of our procedure with an arbitrarily large value of $\eta_{\max }$ leads to disastrous localizations due to numerical instabilities. In fact, with a large fixed value of $\eta_{\max }$, for each $\eta \in\left[-\eta_{\max }, \eta_{\max }\right]^{3}$ such that $\|\eta\|$ is large, the norms of the background potential $E_{0}$ and of the test field $w$ used in (5.5) become too small or too large as compared to their norms for $\|\eta\|$ near 0 . A way to overcome these instabilities is to consider a cutoff process (see, e.g., [22]). For such a process, a threshold $\eta_{\star}$ (independent of the centers and shapes of imperfections as well as of $\mu_{\alpha}, \varepsilon_{\alpha}$ ) is introduced such that for $\|\eta\|>\left\|\left(\eta_{\star}, \eta_{\star}, \eta_{\star}\right)^{T}\right\|$, the quantity in (5.9) is set equal to 0 . We incorporate this process in our procedure by recommending "fine" grids for $\eta$ in order to "compensate" the induced loss of accuracy. In addition to the physical parameters $\mu_{\alpha}, \varepsilon_{\alpha}$, all our numerical experiments will be then described with respect to $\eta_{\max }, n$ and $\eta_{\star}$.

Before presenting the localization results in the context of multiple imperfections $(m>1)$, we want to compare some results of Subsection 6.2 with those that will be obtained here in the context of a single imperfection $(m=1)$. In all the cases we fix $\mu_{0}=\varepsilon_{0}=1$ and the suited values for $\eta_{\star}$ will result from the simulations.

By using most of the values of $\mu_{1}$ and $\varepsilon_{1}$ considered in Subsection 6.2, we represent in Figs. 6.10-6.16 the localization results of a single imperfection, from the settings $\mathcal{T}_{\alpha}^{1}$ and $\mathcal{T}_{\alpha}^{2}$. For each experiment, we fix $\eta_{\max }=10$ and consider $\rho=2$ as the step size for sampling, i.e. $n=10$. The expected order of resolution is then $0.5 \pi / \eta_{\max } \approx 0.157$. This fixed value of $\eta_{\max }$ appears numerically large as observed from simulations, and the mentioned cutoff process is hence required.

The results represented in Figs. 6.10-6.11 show that the localization of the imperfection from $\mathcal{T}_{\alpha}^{1}$ is successfully achieved in both the magnetic and the electromagnetic case, according to the expected accuracy. Similar results have been obtained from simulations based again on $\mathcal{T}_{\alpha}^{1}$, and using the same values of parameters $\eta_{\max }, n, \eta_{\star}$, but by considering $\mu_{1}=10$ with $\varepsilon_{1}=1,5$, and $\mu_{1}=5$ with $\varepsilon_{1}=3$.

As indicate Figs. 6.12-6.16, the localization of a smaller imperfection is also achieved with 

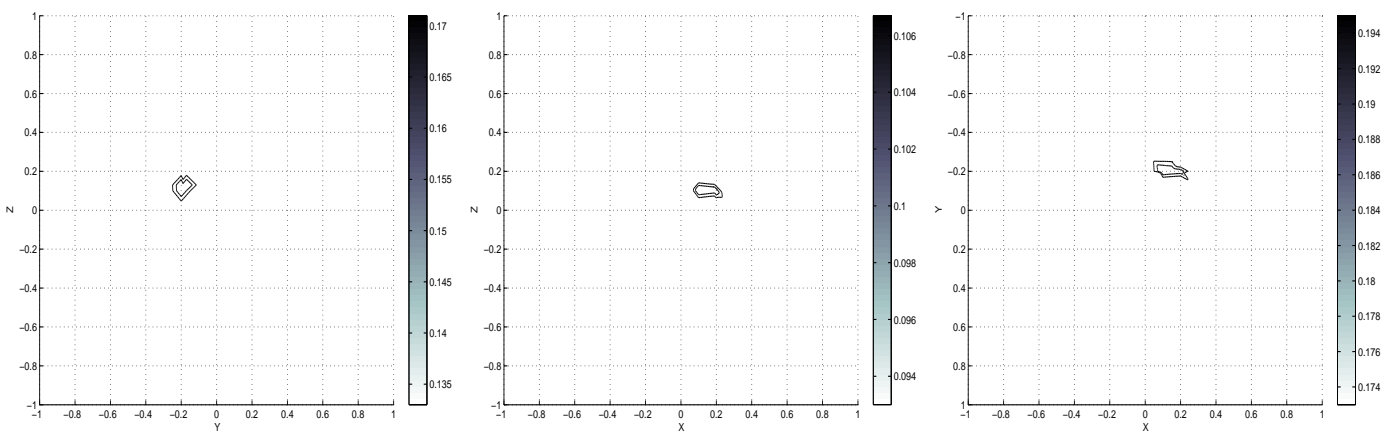

Fig. 6.10. Contour-plot views respectively from the $x$-direction, the $y$-direction and the $z$-direction, based on the enriched sequence, deriving from one of the modules of the terms that approximate those of (5.10). Here, $\mathcal{T}_{\alpha}^{1}$ is used, $\mu_{1}=3, \varepsilon_{1}=1, \eta_{\max }=10, n=10$ and $\eta_{\star}=4.5$.
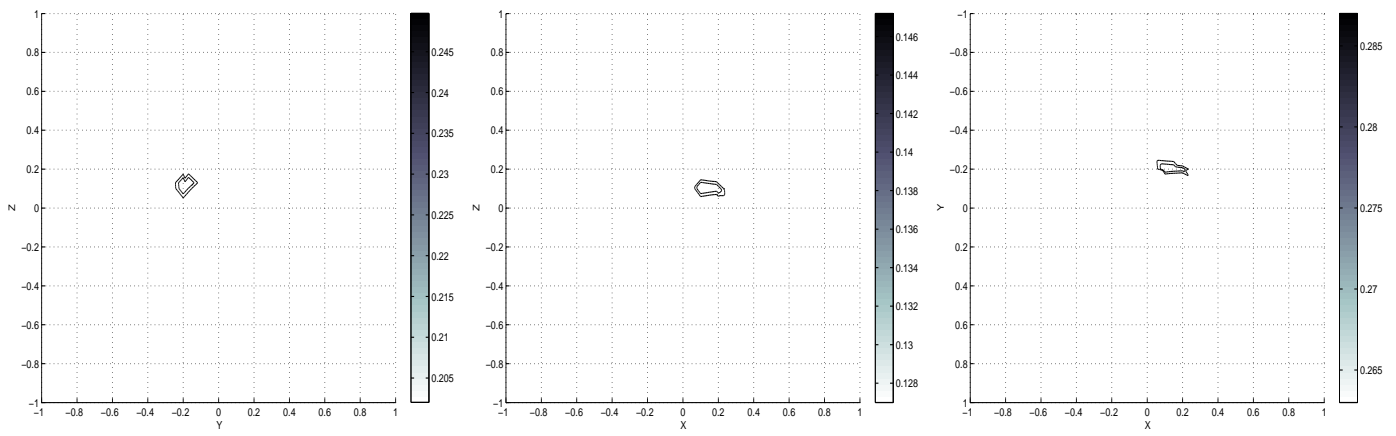

Fig. 6.11. Same as Fig 6.10 except with $\mu_{1}=5, \varepsilon_{1}=10$.

good numerical accuracy, according to the fixed order of resolution. The performed experiments take into account here again different contrasts. Typically, Figs. 6.12-6.13 concern the localization in the magnetic case whereas the results obtained from the same configuration, but in the electromagnetic case, are represented in Figs. 6.14-6.16. As was already the case for the experiments of the previous subsection, we observe from Figs. 6.13-6.14 that the influence of the parameter $\varepsilon_{1}$ is not significant in the localization (the two sequences, whose contour-plot views are represented in these figures, are such that their respective terms have close values). The same observation is also noticed from Figs. 6.15-6.16.

A comparison of these results (see Figs. 6.10-6.12, 6.15), with those of the previous subsection (see Figs. 6.5, 6.7-6.9), allows us to mention, as regards the single imperfection configuration, that the present localization procedure is less efficient than the one based on the Current Projection method. In fact, in contrast with Subsection 6.2, a very large number of numerical measurements is required here (even for an order of resolution which is not very small), and the obtained results are less accurate.

Experiments in the multiple imperfections configuration can be performed here, contrary with the procedure based on the Current Projection method. These experiments will be based here on the settings $\mathcal{T}_{\alpha}^{3}, \mathcal{T}_{\alpha}^{4}$ and $\mathcal{T}_{\alpha}^{5}$. By keeping the same value for $\eta_{\max }$, we expect the same order of resolution as before. We will consider successively $\rho=2, \frac{5}{4}$ as the step size for sampling. The suited values for $\eta_{\star}$ will result again from simulations. In the presentation of our results, when the $z$-direction, for example, will be concerned, the software used for the postprocessing 

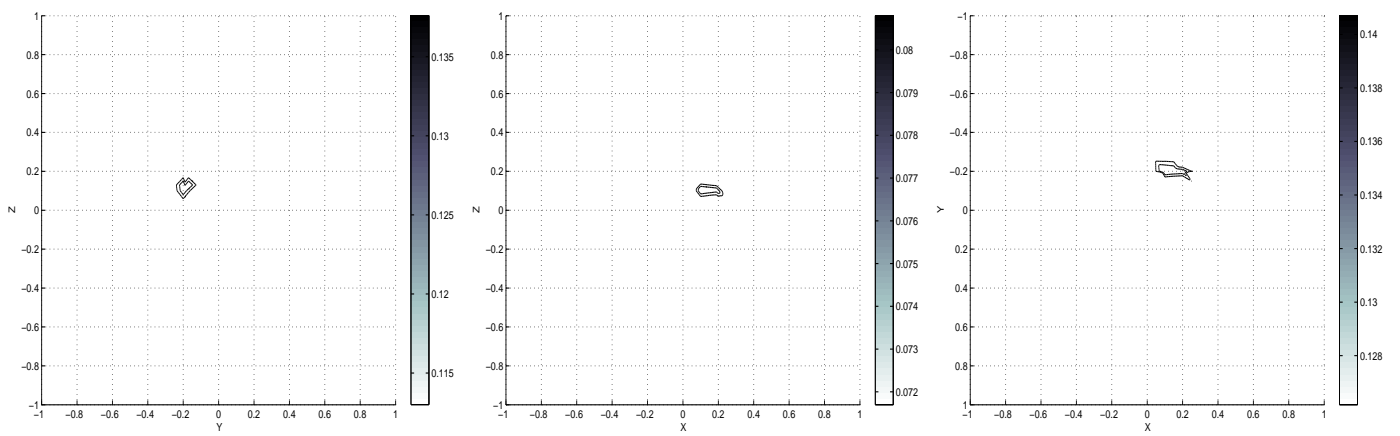

Fig. 6.12. Contour-plot views respectively from the $x$-direction, the $y$-direction and the $z$-direction, based on the enriched sequence, deriving from one of the modules of the terms that approximate those of (5.10). Here, $\mathcal{T}_{\alpha}^{2}$ is used, $\mu_{1}=3, \varepsilon_{1}=1, \eta_{\max }=10, n=10$ and $\eta_{\star}=4.5$.
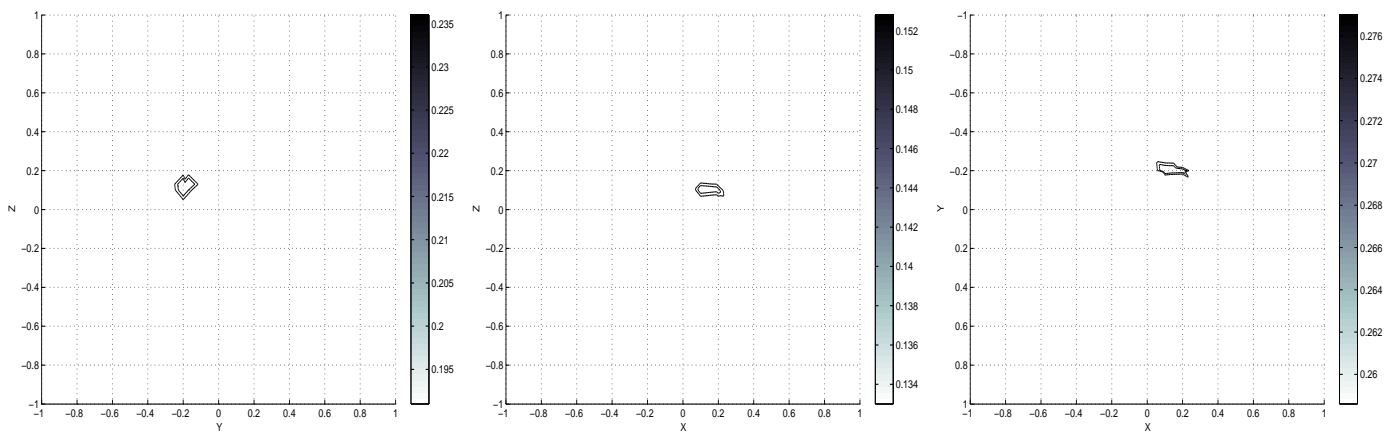

Fig. 6.13. Same as Fig. 6.12 except with $\mu_{1}=10, \varepsilon_{1}=1$.

will draw, besides contour-plot obtained on the plane $O x y$, horizontal and vertical segments whose intersections correspond to centers of the original imperfections viewed on $O x y$.

We represent in Figs. 6.17-6.20 the results of experiments obtained in various aspects, from $\mathcal{T}_{\alpha}^{3}$ and with $\rho=2$. Typically, Fig. 6.17 is associated with the localization of two magnetic imperfections whereas Figs. 6.18-6.20 concern the results of experiments performed with electromagnetic contrasts.

With $\mu_{1}=\mu_{2}=3, \varepsilon_{1}=\varepsilon_{2}=1$, and for the same values of parameters $\eta_{\max }, n$, we have obtained from simulations based again on $\mathcal{T}_{\alpha}^{3}$ a localization similar to the one presented by Fig. 6.17 , but by taking now $\eta_{\star}=4.5$.

The experiment associated with Fig. 6.18 deals with the localization of two imperfections one of which is magnetic and the other electromagnetic.

Contrary to the localization presented by Fig. 6.19, in the case of two electromagnetic imperfections of the same contrast, the one corresponding to Fig. 6.20 concerns electromagnetic imperfections of different contrasts. Similar localizations have been obtained from simulations, based on $\mathcal{T}_{\alpha}^{3}$, by using the same values of parameters $\eta_{\max }, n, \eta_{\star}$, but by considering other contrasts; namely when $\mu_{1}=\mu_{2}=10$ with $\varepsilon_{1}=\varepsilon_{2}=5$, or $\mu_{1}=\mu_{2}=5$ with $\varepsilon_{1}=\varepsilon_{2}=10$, as well as $\mu_{1}=\mu_{2}=5$ with $\varepsilon_{1}=10$, and $\varepsilon_{2}=3$.

The settings $\mathcal{T}_{\alpha}^{1}, \mathcal{T}_{\alpha}^{2}$ and $\mathcal{T}_{\alpha}^{3}$ being associated with a same region of interest $[-K, K]^{3}$ in the procedure and the order of resolution being considered as before, all the experiments have been performed from $\mathcal{T}_{\alpha}^{3}$ with the same number of measurements as in the single imperfection 

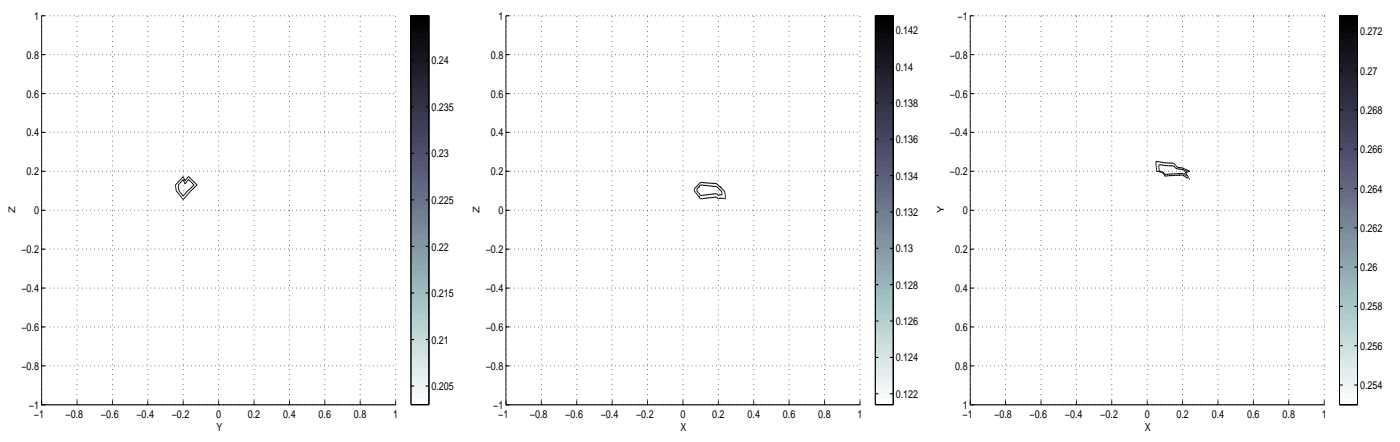

Fig. 6.14. Same as Fig. 6.12 except with $\mu_{1}=10, \varepsilon_{1}=5$.
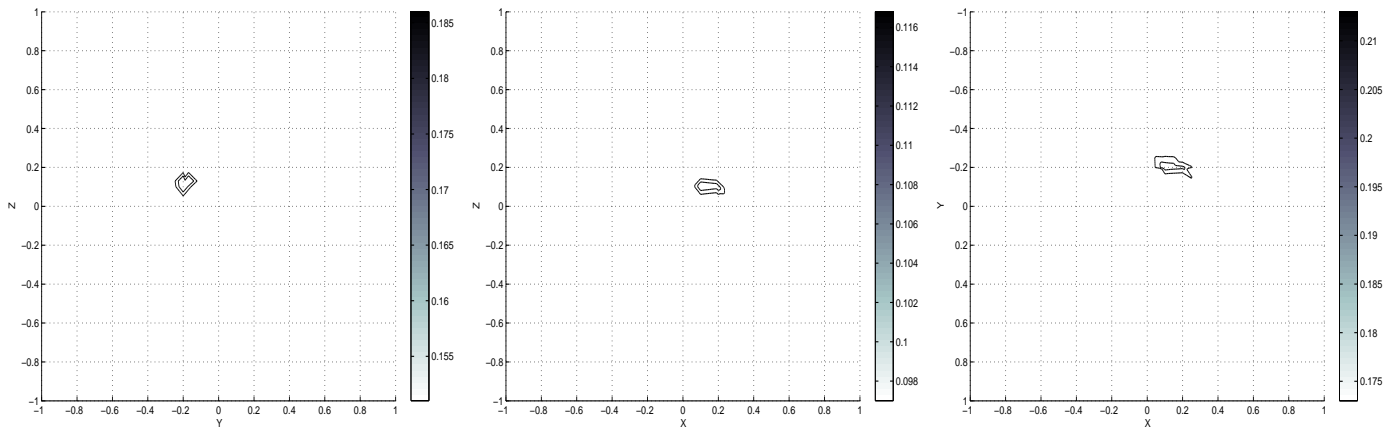

Fig. 6.15. Same as Fig 6.12 except with $\mu_{1}=5, \varepsilon_{1}=3$.
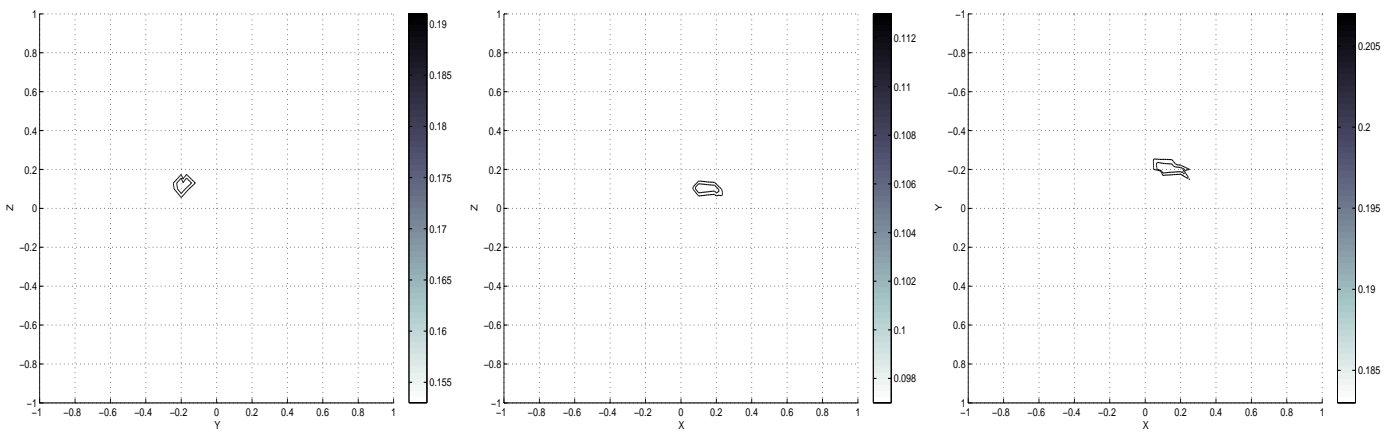

Fig. 6.16. Same as Fig. 6.12 except with $\mu_{1}=5, \varepsilon_{1}=10$.

context. The results of these experiments allow us to notice that the localization based on $\mathcal{T}_{\alpha}^{3}$ is also successfully achieved.

Let us present now the results obtained from experiments based on the settings $\mathcal{T}_{\alpha}^{4}$ and $\mathcal{T}_{\alpha}^{5}$. Since these settings are associated with the same physical region of interest in the procedure, we use a unique value of $\rho$ in simulations: $\rho=5 / 4$. We are hence led to consider a larger number of measurements than in the previous simulations, although keeping a same order of resolution as before.

With a unique choice of values of the parameters $\eta_{\max }, n, \eta_{\star}$, we represent in Figs. 6.21-6.22 the results of the localization of three magnetic imperfections obtained respectively from $\mathcal{T}_{\alpha}^{4}$ and $\mathcal{T}_{\alpha}^{5}$. We already mention that similar localizations are obtained from simulations based on 

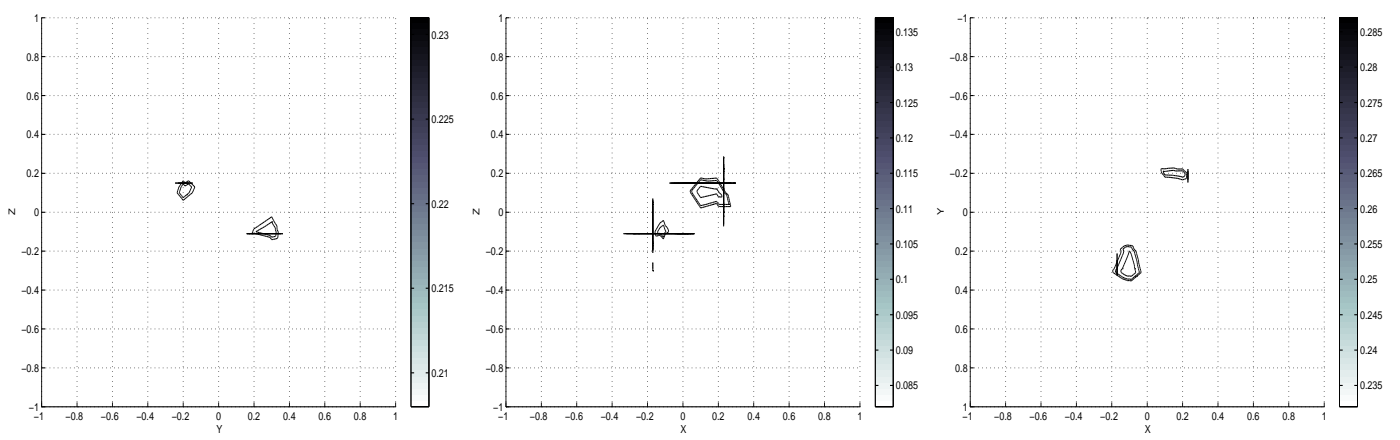

Fig. 6.17. Contour-plot views respectively from the $x$-direction, the $y$-direction and the $z$-direction, based on the enriched sequence, deriving from one of the modules of the terms that approximate those of (5.10). Here, $\mathcal{T}_{\alpha}^{3}$ is used, $\mu_{1}=\mu_{2}=10, \varepsilon_{1}=\varepsilon_{2}=1, \eta_{\max }=10, n=10$ and $\eta_{\star}=4$.
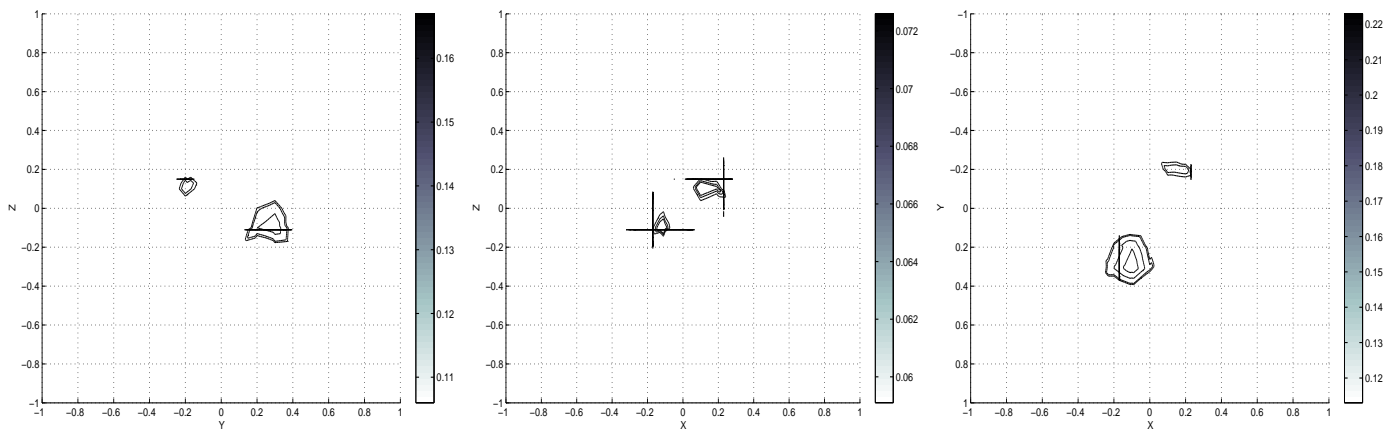

Fig. 6.18. Same as Fig. 6.17 except with $\mu_{1}=5, \mu_{2}=3, \varepsilon_{1}=3, \varepsilon_{2}=1$.
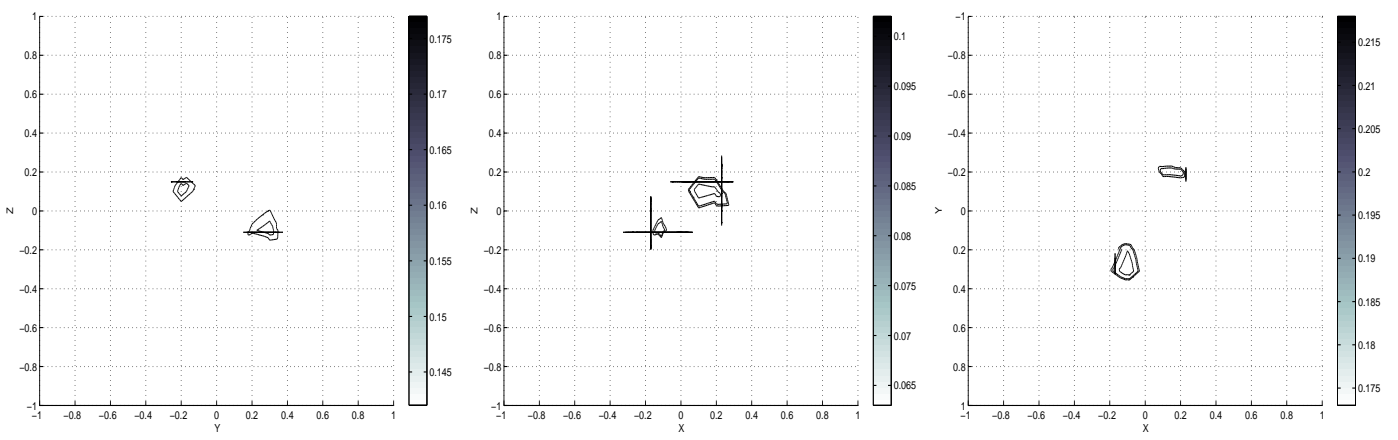

Fig. 6.19. Same as Fig. 6.17 except with $\mu_{1}=\mu_{2}=5, \varepsilon_{1}=\varepsilon_{2}=3$.

these settings and for the same values of parameters but by considering $\mu_{j}=3$ with $\varepsilon_{j}=1$, $1 \leq j \leq 3$. The accuracy of the localization from $\mathcal{T}_{\alpha}^{4}$ is similar to the one resulting from $\mathcal{T}_{\alpha}^{5}$.

The experiment associated with Fig. 6.23, and performed from $\mathcal{T}_{\alpha}^{4}$, concerns the localization of three imperfections where one is purely magnetic and the other ones are electromagnetic.

As opposed to the choice of a unique physical contrast in the localization of imperfections, presented by Fig. 6.24, the experiment associated with Fig. 6.25 concerns also electromagnetic imperfections but with physical contrasts that are different. A similar localization to the one presented by Fig. 6.24 is obtained from simulations based on $\mathcal{T}_{\alpha}^{5}$ and the same values of $\eta_{\max }$, $n, \eta_{\star}$, but in the case of a lower contrast, namely with $\mu_{j}=5, \varepsilon_{j}=1,1 \leq j \leq 3$. 

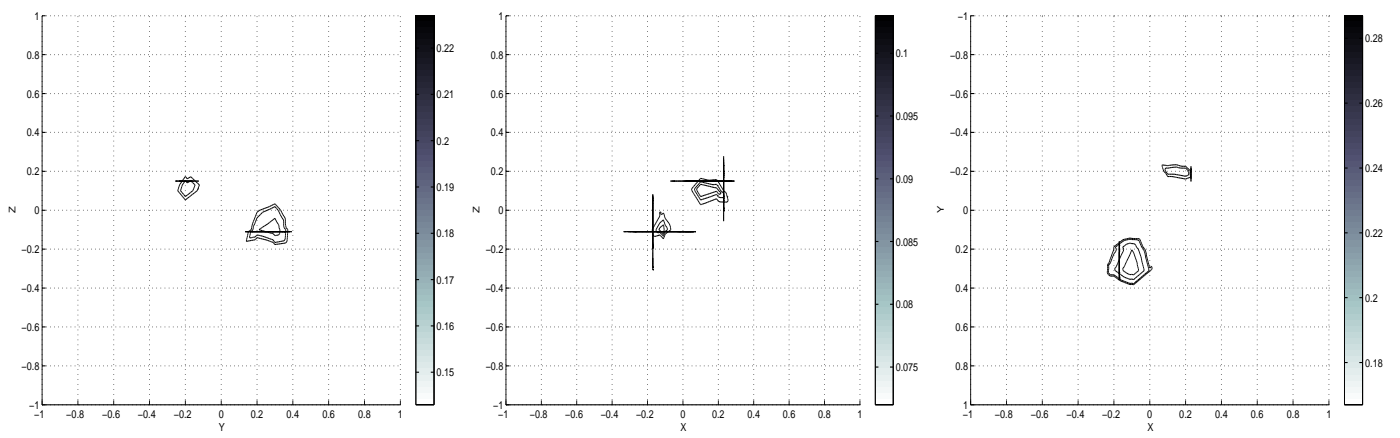

Fig. 6.20. Same as Fig. 6.17 except with $\mu_{1}=10, \mu_{2}=5, \varepsilon_{1}=5, \varepsilon_{2}=10$.
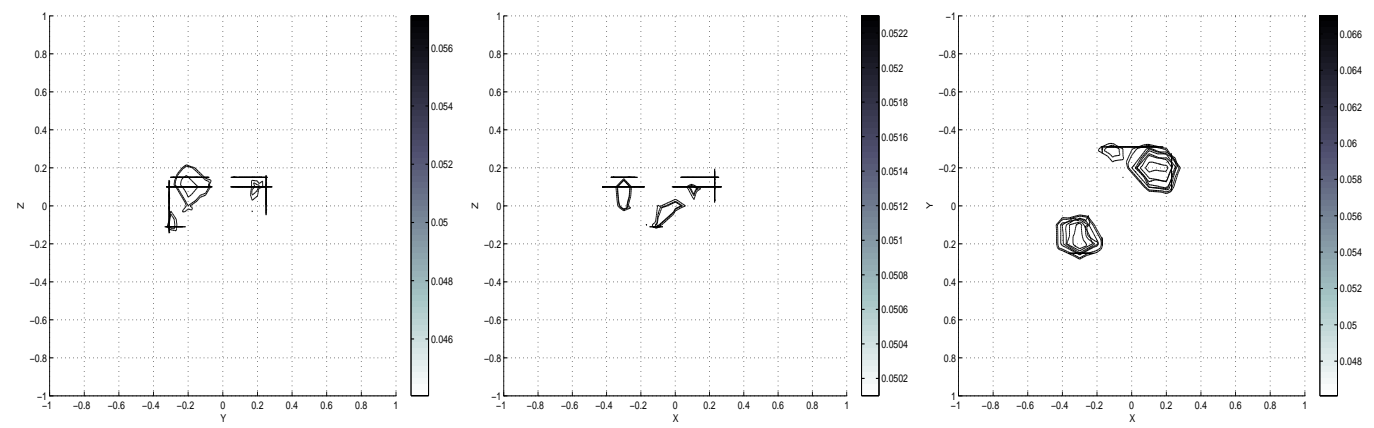

Fig. 6.21. Contour-plot views respectively from the $x$-direction, the $y$-direction and the $z$-direction, based on the enriched sequence, deriving from one of the modules of the terms that approximate those of (5.10). Here, $\mathcal{T}_{\alpha}^{4}$ is used, $\mu_{j}=10, \varepsilon_{j}=1(1 \leq j \leq 3), \eta_{\max }=10, n=16$ and $\eta_{\star}=3$.
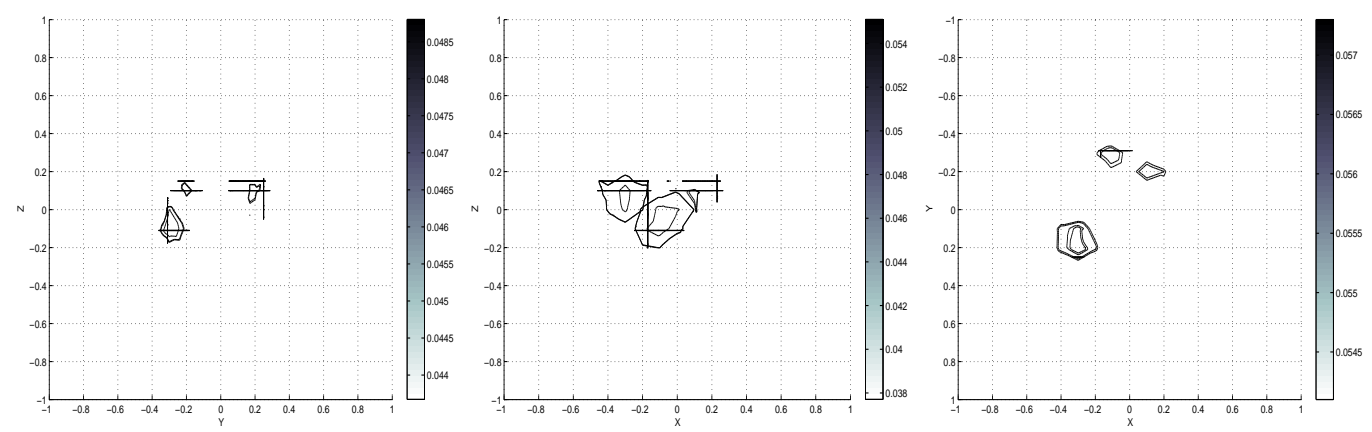

Fig. 6.22. Contour-plot views respectively from the $x$-direction, the $y$-direction and the $z$-direction, based on the enriched sequence, deriving from one of the modules of the terms that approximate those of (5.10). Here, $\mathcal{T}_{\alpha}^{5}$ is used, $\mu_{j}=10, \varepsilon_{j}=1(1 \leq j \leq 3), \eta_{\max }=10, n=16$ and $\eta_{\star}=3$.

According to the fixed order of resolution, an inspection of the results of Figs. 6.21-6.25 shows that the localization from $\mathcal{T}_{\alpha}^{4}, \mathcal{T}_{\alpha}^{5}$ is also achieved with good numerical accuracy.

In order to improve the localization accuracy from the present procedure, we must consider very large values for $\eta_{\max }$. Nevertheless, with a very large value of $\eta_{\max }$, we are concerned in the procedure with a number of measurements which, despite the cutoff process of the Fourier domain, remains very large and amplifies the localization CPU time - for instance, 305 measurements are actually evaluated in the case of the experiment associated with Fig. 6.25. 

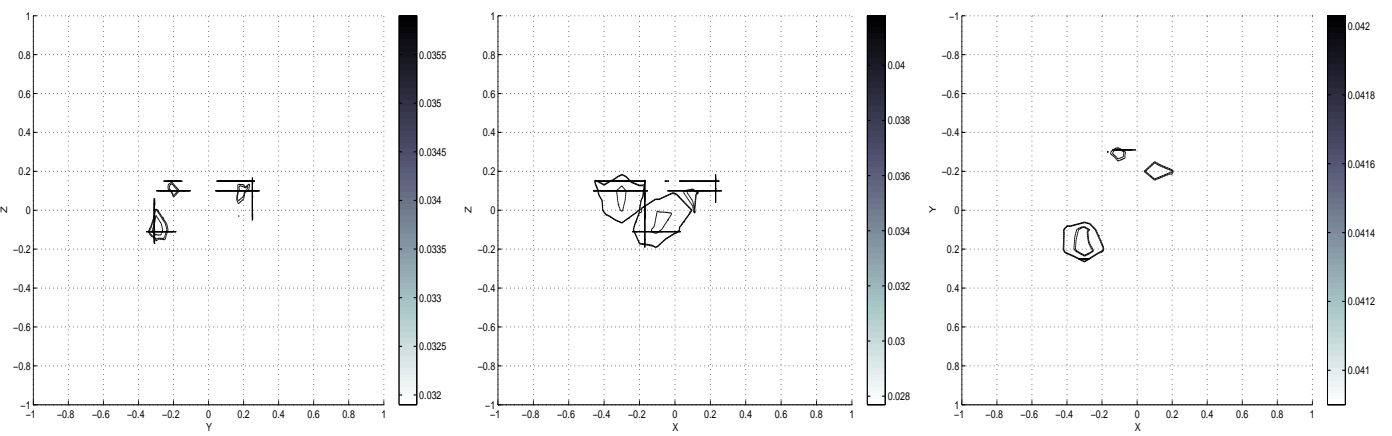

Fig. 6.23. Same as Fig. 6.21 except with $\mu_{1}=\mu_{2}=5, \mu_{3}=3, \varepsilon_{1}=\varepsilon_{2}=3, \varepsilon_{3}=1$.
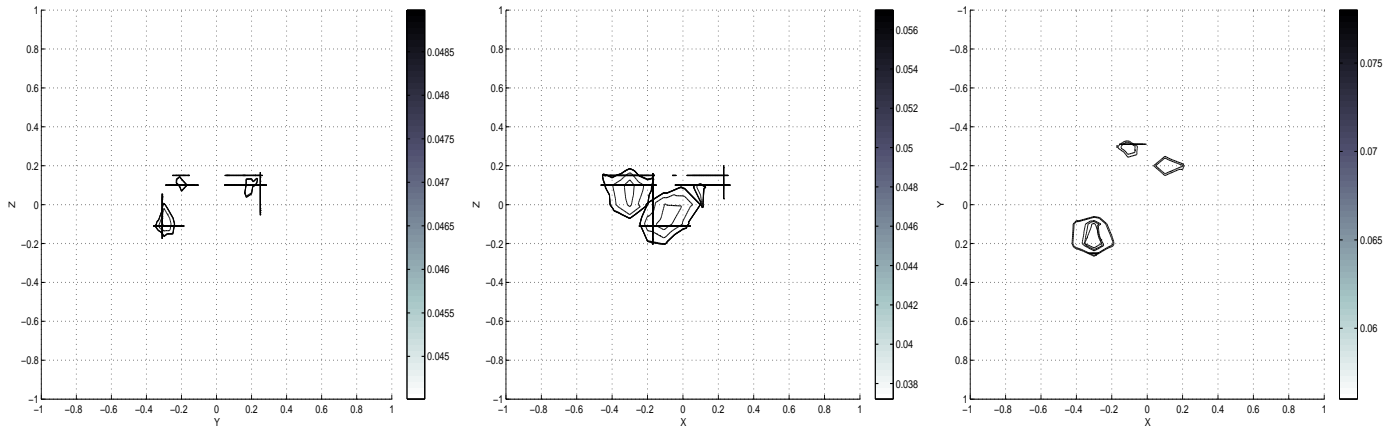

Fig. 6.24. Same as Fig. 6.22 except with $\mu_{j}=10, \varepsilon_{j}=3(1 \leq j \leq 3)$.
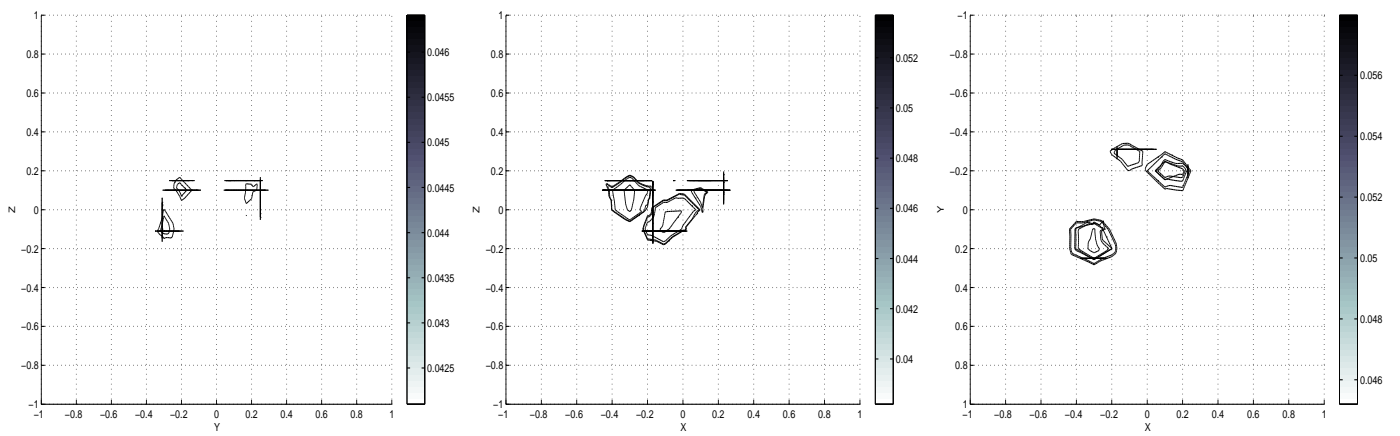

Fig. 6.25. Same as Fig. 6.21 except with $\mu_{1}=\mu_{2}=10, \mu_{3}=5, \varepsilon_{1}=\varepsilon_{2}=5, \varepsilon_{3}=10$.

By taking into account moreover the fact that the evaluation of each measurement, related to computations based on a full mesh of the domain, has a relatively important cost - average CPU time of about 15.840s. on an "IBM Processor P575 with a frequency of $1.9 \mathrm{GHz}$ ", in the case of $\mathcal{T}_{\alpha}^{4}$ for instance, and without adding the CPU time for evaluating the right-hand side of (4.3) associated with the measurement, it follows with such a value of $\eta_{\max }$ that the localization can only be achieved with an exorbitant CPU time. Typically, the CPU time is the main disadvantage of this procedure; it remains in fact expensive when compared for instance with the one needed by the procedure based on the Current Projection method in the single imperfection context, because of the large number of measurements required even for a value of $\eta_{\max }$ which is not, as in the previous experiments, very large. 


\section{Conclusions}

We have achieved the numerical localization of certain electromagnetic imperfections, in a three-dimensional bounded domain, from a limit perturbation model in the tangential boundary trace of the curl of the electric field, associated with a limit model in electric field introduced by letting the frequency vanish in the time-harmonic Maxwell equations. The experiments were performed from two localization procedures, introduced by combining the limit perturbation model with a Current Projection method or, distinctly, with an Inverse Fourier method.

The obtained numerical results lead us to answer positively the question set in the introduction of this paper: the present localization framework is well-suited for imperfections that are not purely electric.

Here, the experiments performed both in the single imperfection context as well as in the multiple imperfections configuration do not deal with the purely electric case. In order to localize also purely electric imperfections from the limit model in electric field, it is necessary to build another boundary perturbation formula that contains now information with regard to the electric permittivity. We think for example of a perturbation formula in the tangential boundary trace of the curl of the electric field, introduced directly from (3.2) with the help of test vector fields $w$ that are not as in (3.11), of constant divergence.

It is observed from experiments that the procedure using a Current Projection method is more suitable than the one based on an Inverse Fourier method, in the single imperfection case. Accurate localizations having to be obtained from the procedure based on an Inverse Fourier method by taking in particular increasing numbers of boundary measurements, it results that this procedure is very expensive in CPU time. In fact, associated with a prescribed boundary electric current, each measurement is numerically obtained from the computation, for this prescribed datum, of the solution of the discrete formulation (4.3) whereas the CPU time needed to solve (4.3) is already relatively important. Typically, the rectangular system resulting from (4.3) has a large number of scalar unknowns caused by the smallness of the imperfections since a full conforming mesh of the three-dimensional domain $\Omega$ is considered, and moreover mixed finite elements are used; even by applying a preconditioning technique, as done here, the CPU time required for determining this solution remains relatively important. Although the procedure based on an Inverse Fourier method seems well-suited to the context of a large number of imperfections contained in the domain, we did not perform here simulations in this context since the diameters of such imperfections should be, according to our present configuration hypotheses, much smaller than those considered here, and the size of the rectangular system resulting from (4.3) would be in that case too large - prohibiting thus flexible numerical investigations because of the excessive memory storage required. In such a context, we must modify our localization procedures by achieving the stage of the numerical evaluation of boundary measurements with the help of integral equations techniques for instance.

Acknowledgments. This work was partly supported by ACI NIM (171) from the French Ministry of Education and Scientific Research.

\section{References}

[1] H. Ammari, E. Iakovleva and D. Lesselier, A MUSIC algorithm for locating small inclusions buried in a half-space from the scattering amplitude at a fixed frequency, Multiscale Model. Sim., 3 (2005), 597-628. 
[2] H. Ammari and H. Kang, Reconstruction of Small Inhomogeneities from Boundary Measurements, Lecture Notes in Mathematics, v. 1846, Springer-Verlag, Berlin, 2004.

[3] H. Ammari and A. Khelifi, Electromagnetic scattering by small dielectric inhomogeneities, $J$. Math. Pures Appl., 82 (2003), 749-842.

[4] H. Ammari, S. Moskow and M.S. Vogelius, Boundary integral formulas for the reconstruction of electromagnetic imperfections of small diameter, ESAIM Control Optim. Calc. Var., 9 (2003), 49-66.

[5] H. Ammari, M. Vogelius and D. Volkov, Asymptotic formulas for perturbations in the electromagnetic fields due to the presence of imperfections of small diameter II. The full Maxwell equations, J. Math. Pures Appl., 80 (2001), 769-814.

[6] H. Ammari and D. Volkov, Asymptotic formulas for perturbations in the eigenfrequencies of the full Maxwell equations due to the presence of imperfections of small diameter, Asymptotic. Anal., 30 (2002), 331-350.

[7] M. Asch and S.M. Mefire, Numerical localization of electromagnetic imperfections from a perturbation formula in three dimensions, J. Comput. Math., 26 (2008), 149-195.

[8] M. Brühl and M. Hanke, Numerical implementation of two noniterative methods for locating inclusions by impedance tomography, Inverse Probl., 16 (2000), 1029-1042.

[9] A.P. Calderón, On an inverse boundary value problem, Seminar on Numerical Analysis and its Applications to Continuum Physics, Soc. Brasileira de Matemática, Rio de Janeiro, (1980), 65-73.

[10] D.J. Cedio-Fengya, S. Moskow and M.S. Vogelius, Identification of conductivity imperfections of small diameter by boundary measurements. Continuous dependence and computational reconstruction, Inverse Probl., 14 (1998), 553-595.

[11] D. Colton and R. Kress, Integral Equation Methods in Scattering Theory, Krieger Publ. Co., Malabar, Florida, 1992.

[12] D.C. Dobson and F. Santosa, An image-enhancement technique for electrical impedance tomography, Inverse Probl., 10 (1994), 317-334.

[13] G.B. Folland, Introduction to Partial Differential Equations, Princeton University Press, Princeton, 1976.

[14] P. Joly and G. Meurant, Complex conjugate gradient methods, Numer. Algorithms, 4 (1993), 379-406.

[15] A. Kirsch, The MUSIC algorithm and the factorization method in inverse scattering theory for inhomogeneous media, Inverse Probl., 18 (2002), 1025-1040.

[16] R.V. Kohn and A. McKenny, Numerical implementation of a variational method for electrical impedance tomography, Inverse Probl., 6 (1990), 389-414.

[17] J. Laminie and S.M. Mefire, Three-dimensional computation of a magnetic field by mixed finite elements and boundary elements, Appl. Numer. Math. 35 (2000), 221-244.

[18] J.-C. Nédélec, Mixed finite elements in $\mathbb{R}^{3}$, Numer. Math., 35 (1980), 315-341.

[19] J.-C. Nédélec, Elements Finis mixtes incompressibles pour l'équation de Stokes dans $\mathbb{R}^{3}$, Numer. Math., 39 (1982), 97-112.

[20] F. Santosa and M.S. Vogelius, A backprojection algorithm for electrical impedance imaging, SIAM J. Appl. Math., 50 (1990), 216-243.

[21] E. Somersalo, M. Cheney, D. Isaacson and E. Isaacson, Layer-stripping: a direct numerical method for impedance imaging, Inverse Probl., 7 (1991), 899-926.

[22] D. Volkov, Numerical methods for locating small dielectric inhomogeneities, Wave Motion, 38 (2003), 189-206.

[23] L.T. Yang, Accuracy of preconditioned CG-type methods for least squares problems, Comput. Math. Appl., 45 (2003), 77-96. 